\title{
LAGUERRE EXPANSIONS ON CONIC DOMAINS
}

\author{
YUAN XU
}

\begin{abstract}
We study the Fourier orthogonal expansions with respect to the Laguerre type weigh functions on the conic surface of revolution and the domain bounded by such a surface. The main results include a closed form formula for the reproducing kernels of the orthogonal projection operator and a pseudo convolution structure on the conic domain; the latter is shown to be bounded in an appropriate $L^{p}$ space and used to study mean convergence of the Cesàro means of the Laguerre expansions on conic domains.
\end{abstract}

\section{INTRODUCTION}

The Laguerre expansions usually mean the Fourier orthogonal expansions in the Laguerre polynomials on the half-line $\mathbb{R}_{+}=[0, \infty)$ or expansions in product Laguerre polynomials on $\mathbb{R}_{+}^{d}$ for $d \geq 2$ (cf. [18). In the present paper, we study the Fourier orthogonal expansions on the conic surface

$$
\mathbb{V}_{0}^{d+1}=\left\{(x, t):\|x\|=t, \quad x \in \mathbb{R}^{d}, t \in \mathbb{R}_{+}\right\}
$$

with respect to the polynomials that are orthogonal with respect to the weight function

$$
\mathrm{w}_{\kappa}(x, t)=h_{\kappa}^{2}(x) t^{-1} \mathrm{e}^{-t} \quad \text { with } \quad h_{\kappa}(x)=\prod_{k=1}^{d}\left|x_{i}\right|^{\kappa_{i}}, \quad(x, t) \in \mathbb{V}_{0}^{d+1},
$$

as well as the expansions on the solid cone $\mathbb{V}^{d+1}$ bounded by the surface $\mathbb{V}_{0}^{d+1}$. This is the first study for the Laguerre type weight on conic domains; our results are new even for $\mathrm{w}_{0}(x, t)=t^{-1} e^{-t}$.

If $f(x, t)=f_{0}(t)$ with $f_{0}: \mathbb{R}_{+} \mapsto \mathbb{R}$, then the orthogonal expansions of $f$ on the conic domain becomes the classical Laguerre expansions on $\mathbb{R}_{+}$, which has been studied extensively in the literature; see [1, 7, 8, 9, 10, 14, 17, 18, and, for some more recent works, see 2, 11, 12, 15, and the references therein. As in the study of classical Laguerre expansions, our study relies on intrinsic properties of orthogonal polynomials that hold only for particular weight functions and special domains. The study of orthogonal structure on the conic domains is initiated recently in [22, 23]. A basis of orthogonal polynomials with respect to $\mathrm{w}_{\kappa}$, called the Laguerre type polynomials below, can be given in terms of the classical Laguerre polynomials and spherical $h$-harmonics, the latter are orthogonal polynomials with respect to $h_{\kappa}^{2}(x)$ on the unit sphere $\mathbb{S}^{d-1}$ and are the simplest examples in the Dunkl's theory of $h$-harmonics associated with reflection groups. This basis is briefly treated in [22, where the main results are established for the Jacobi type polynomials on compact conic domains, for which $t \in \mathbb{R}_{+}$is replace by $t \in[0,1]$ and $\mathrm{e}^{-t}$ is replace by $(1-t)^{\gamma}$. Our starting point is to show that the

Date: March 9, 2021.

2010 Mathematics Subject Classification. 33C50, 35C10, 42C05, 42C10.

Key words and phrases. Laguerre polynomials, orthogonal expansions, convolution operator, conic domains. 
Laguerre type polynomials with respect to $w_{\kappa}$ on the conic surface can be regarded as limits of the Jacobi type polynomials on the compact conic surface, which allows us to derive properties of the former from those of the latter. In particular, starting from the closed formula for the reproducing kernels of the Jacobi type polynomials in 22], we are able to deduce closed form formulas for the Poisson kernel and for the reproducing kernels of the Laguerre type polynomials on the conic domains. Since the reproducing kernels are kernels of the orthogonal projection operators, the closed form formula provides an essential tool for our analysis. It leads to the definition of a pseudo convolution structure on the conic surface, defined via a generalized translation operator, which is shown to be bounded in an appropriate $L^{p}$ space. As an application, we use the convolution structure to study the mean convergence of the Cesàro $(C, \delta)$ means of the Laguerre expansions on the conic surface and determine the critical index for the convergence. We will also establish analogues results on the solid cone $\mathbb{V}^{d+1}$ with respect to the weight function $W_{\kappa, \mu}(x, t)=h_{\kappa}^{2}(x)\left(t^{2}-\|x\|^{2}\right)^{\mu-\frac{1}{2}} \mathrm{e}^{-t}$, which can be deduced from a closed relation between the orthogonal structures on the two domains, akin to the relation between the unit ball and the unit sphere [6].

The above narrative of our main results on the conic surface is rooted in the study of the Laguerre expansions on $\mathbb{R}_{+}^{d}$. The difference in the domain, however, means different geometry and different obstacles. The closed form formula for the reproducing kernel, hence the generalized translation operator, is substantially more involved on the conic domains. Because of the complexity of the closed form formula, proving the boundedness of the pseudo convolution becomes far more elaborate. There is also a hidden subtle dependence on the parameters, which shows, in the case of $w_{0}$, that the convolution has a weaker upper bound for $d=2$ than that for $d \geq 3$. The analysis on the conic surface is not a straightforward extension of the ordinary Laguerre expansions.

The paper is organized as follows. In the next section we collect results on classical orthogonal polynomials and special functions that will be needed. The Laguerre type polynomials and the closed form formulas for the Poisson and reproducing kernels will be studied in the the third section. The generalized translation operator and the pseudo convolution will be defined in the fourth section, where they are shown to be bounded and used to study the Cesàro means. Finally, the results on the conic surface are extended to solid cone in the fifth section.

\section{Preliminary}

We recall several families of orthogonal polynomials that will be needed in this section. These are the Laguerre polynomials, the Gegenbauer and Jacobi polynomials, and spherical $h$-harmonics on the unit sphere and orthogonal polynomials on the unit ball.

2.1. Laguerre polynomials. For $\alpha>-1$, the Laguerre polynomials $L_{n}^{\alpha}$ are given by

$$
L_{n}^{\alpha}(t)=\frac{(\alpha+1)_{n}}{n !} \sum_{k=0}^{n} \frac{(-n)_{k}}{k !(\alpha+1)_{k}} t^{k},
$$

and they can also be defined by their generating function

$$
\sum_{n=0}^{\infty} L_{n}^{\alpha}(t) r^{n}=\frac{1}{(1-r)^{\alpha+1}} \mathrm{e}^{-\frac{t r}{1-r}}
$$


The Laguerre polynomials are orthogonal with respect to the inner product

$$
\langle f, g\rangle_{\alpha}=b_{\alpha} \int_{0}^{\infty} f(t) g(t) w_{\alpha}(t) \mathrm{d} t, \quad w_{\alpha}(t):=t^{\alpha} e^{-t}, \quad b_{\alpha}:=\frac{1}{\Gamma(\alpha+1)},
$$

which is normalized so that $\langle 1,1\rangle_{\alpha}=1$. More precisely, for $n, m \in \mathbb{N}_{0}$,

$$
\left\langle L_{n}^{\alpha}, L_{m}^{\alpha}\right\rangle_{\alpha}=h_{n}^{\alpha} \delta_{n, m}, \quad \text { where } \quad h_{n}^{\alpha}:=\frac{(\alpha+1)_{n}}{n !}=L_{n}^{\alpha}(0) .
$$

For $f \in L^{2}\left(\mathbb{R}_{+}, w_{\alpha}\right)$, its Fourier-Laguerre expansion is defined by

$$
f(t)=\sum_{n=0}^{\infty} \widehat{f}_{n}^{\alpha} \frac{L_{n}^{\alpha}(t)}{\sqrt{h_{n}^{\alpha}}}, \quad \text { where } \quad \widehat{f}_{n}^{\alpha}=\frac{1}{\sqrt{h_{n}^{\alpha}}}\left\langle f, L_{n}^{\alpha}\right\rangle_{\alpha} .
$$

One of the important tool for studying the Laguerre expansions is a convolution structure motivated by the product formula of the Laguerre polynomials. Let $J_{\alpha}$ be the usual Bessel function and let $j_{\alpha}(z):=z^{-\alpha} J_{\alpha}(z)$. For $\alpha>-\frac{1}{2}$, the product formula is classical [19]; see also [18, p. 142],

$$
\begin{aligned}
\frac{L_{n}^{\alpha}(t) L_{n}^{\alpha}(s)}{L_{n}^{\alpha}(0)}= & \frac{2^{\alpha} \Gamma(\alpha+1)}{\sqrt{2 \pi}} \int_{0}^{\pi} L_{n}^{\alpha}(t+s+2 \sqrt{t s} \cos \theta) \\
& \times \mathrm{e}^{-\sqrt{t s} \cos \theta} j_{\alpha-\frac{1}{2}}(\sqrt{t s} \sin \theta)(\sin \theta)^{2 \alpha} \mathrm{d} \theta,
\end{aligned}
$$

whereas for $\alpha=-\frac{1}{2}$, the formula is given in [8] and attributed to J. Boersma,

$$
\begin{aligned}
& \frac{L_{n}^{-\frac{1}{2}}(t) L_{n}^{-\frac{1}{2}}(s)}{L_{n}^{-\frac{1}{2}}(0)}=\frac{1}{2}\left[\mathrm{e}^{-\sqrt{t s}} L_{n}^{-\frac{1}{2}}(t+s+2 \sqrt{t s})+\mathrm{e}^{\sqrt{t s}} L_{n}^{-\frac{1}{2}}(t+s-2 \sqrt{t s})\right] \\
& \quad-t s \int_{0}^{\pi} L_{n}^{-\frac{1}{2}}(t+s+2 \sqrt{t s} \cos \theta) \mathrm{e}^{-\sqrt{t s} \cos \theta} j_{1}(\sqrt{t s} \sin \theta) \sin \theta \mathrm{d} \theta .
\end{aligned}
$$

The convolution is defined as an integral operator with the kernel $T_{t} g(s)$, where $T_{t}$ is defined by $T_{t} L_{n}^{\alpha}(s)=L_{n}^{\alpha}(t) L_{n}^{\alpha}(s) / L_{n}^{\alpha}(0)$ for all $n \geq 0$. The convolution is bounded in the space $L_{\alpha}^{p}\left(\mathbb{R}_{+}\right)$that has the norm defined by 7$]$

$$
\|g\|_{p, u(\alpha)}=\left(b_{\alpha} \int_{\mathbb{R}_{+}}\left|g(t) \mathrm{e}^{-t / 2}\right|^{p} t^{\alpha} \mathrm{d} t\right)^{\frac{1}{p}}, \quad 1 \leq p<\infty,
$$

where $b_{\alpha}=1 / \Gamma(\alpha+1)$ and, for $p=\infty$,

$$
\|g\|_{\infty}=\|g\|_{\infty, u(\alpha)}:=\operatorname{ess} \sup \left\{|g(t)| \mathrm{e}^{-t / 2}: t>0\right\} .
$$

For $p=2$, the norm $\|\cdot\|_{2, u(\alpha)}$ coincides with the usual norm of $L^{2}\left(\mathbb{R}_{+}, w_{\alpha}\right)$.

For the summability of the Laguerre expansions, one often considers the Cesàro mans. For $\delta \in \mathbb{R}$, the Cesàro means of the sequence $\left\{a_{n}\right\}_{n=0}^{\infty}$ are defeind by

$$
s_{n}^{\delta}=\frac{1}{A_{n}^{\delta}} \sum_{k=0}^{n} A_{n-k}^{\delta} a_{k}, \quad A_{n}^{\delta}=\left(\begin{array}{c}
n+\delta \\
n
\end{array}\right), \quad n=1,2, \ldots,
$$

which can also be defined by the relation

$$
\frac{1}{(1-r)^{\delta+1}} \sum_{n=0}^{\infty} a_{n} r^{n}=\sum_{n=0}^{\infty} A_{n}^{\delta} s_{n}^{\delta} r^{n} .
$$


We denote the $(C, \delta)$ means of the Fourier-Laguerre series by $s_{n}^{\delta}\left(w_{\alpha} ; f\right)$. The case $\delta=0$ is the partial sum operator, which we denote by $s_{n}\left(\varpi_{\alpha} ; f\right)$; that is,

$$
s_{n}\left(w_{\alpha} ; f\right)=\sum_{k=0}^{n} f_{n}^{\alpha} L_{n}^{\alpha} \quad \text { and } \quad s_{n}^{\delta}\left(w_{\alpha} ; f\right)=\frac{1}{A_{n}^{\delta}} \sum_{k=0}^{n} A_{n-k}^{\delta} f_{k}^{\alpha} L_{k}^{\alpha} .
$$

For $\alpha>-1$, the Poisson kernel of the Laguerre polynomials satisfies

$$
\sum_{n=0}^{\infty} \frac{L_{n}^{\alpha}(x) L_{n}^{\alpha}(y)}{\left(\begin{array}{c}
n+\alpha \\
n
\end{array}\right)} r^{n}=\frac{\Gamma(\alpha+1)}{(1-r)^{\alpha+1}} \mathrm{e}^{-\frac{(x+y) r}{1-r}}(x y r)^{-\frac{\alpha}{2}} I_{\alpha}\left(\frac{2 \sqrt{x y r}}{1-r}\right),
$$

where $I_{\alpha}(z)=e^{-\mathrm{i} \alpha \pi / 2} J_{\alpha}\left(z e^{\mathrm{i} \pi / 2}\right)$ is the modified Bessel function of the first kind [3, (10.27.6)]. For $\alpha \geq-\frac{1}{2}, I_{\alpha}$ has an integral representation [3, (10.32.4)], which leads to the following formula for the Poisson kernel: for $\alpha \geq-\frac{1}{2}$,

$$
\sum_{n=0}^{\infty} \frac{L_{n}^{\alpha}(x) L_{n}^{\alpha}(y)}{\left(\begin{array}{c}
n+\alpha \\
n
\end{array}\right)} r^{n}=\frac{1}{(1-r)^{\alpha+1}} \mathrm{e}^{-\frac{(x+y) r}{1-r}} c_{\alpha} \int_{-1}^{1} \mathrm{e}^{\frac{2 \sqrt{x y r}}{1-r}}\left(1-v^{2}\right)^{\alpha-\frac{1}{2}} \mathrm{~d} v,
$$

where the constant $c_{\alpha}$ is given by

$$
c_{\alpha}=\frac{\Gamma(\alpha+1)}{\Gamma\left(\frac{1}{2}\right) \Gamma\left(\alpha+\frac{1}{2}\right)}, \quad \alpha>-\frac{1}{2},
$$

and the formula holds under the limit for $\alpha=-\frac{1}{2}$ using

$$
\lim _{a \rightarrow \frac{1}{2}+} c_{\alpha} \int_{-1}^{1} f(t)\left(1-t^{2}\right)^{a-\frac{1}{2}} \mathrm{~d} t=\frac{f(1)+f(-1)}{2} .
$$

Finally, we will need the following lemma [8, Lemma 1] or [18, Lemma 1.5.4].

Lemma 2.1. Let $\alpha+\beta>-1$. Then

$$
b_{\alpha} \int_{0}^{\infty}\left|L_{n}^{\alpha+\beta}(t)\right| t^{\alpha / 2} \mathrm{e}^{-t / 2} \mathrm{~d} t \sim \begin{cases}n^{\frac{\alpha+1}{2}}, & \text { if } \beta<\frac{3}{2} \\ n^{\frac{\alpha+1}{2}} \log n, & \text { if } \beta=\frac{3}{2} \\ n^{\frac{\alpha}{2}+\beta-1}, & \text { if } \beta>\frac{3}{2} .\end{cases}
$$

2.2. Jacobi and Gegenbauer Jacobi polynomials. We will also need the Jacobi polynomials, denoted by $P_{n}^{(\alpha, \beta)}$, that satisfy the orthogonal relation

$$
c_{\alpha, \beta}^{\prime} \int_{-1}^{1} P_{n}^{(\alpha, \beta)}(t) P_{m}^{(\alpha, \beta)}(t)(1-t)^{\alpha}(1+t)^{\beta} \mathrm{d} t=h_{n}^{(\alpha, \beta)} \delta_{n, m}, \quad \alpha, \beta>-1,
$$

where the normalization constant $c_{\alpha, \beta}^{\prime}=2^{-\alpha-\beta-1} c_{\alpha, \beta}$ with

$$
c_{\alpha, \beta}=\frac{\Gamma(\alpha+\beta+2)}{\Gamma(\alpha+1) \Gamma(\beta+1)}
$$

and $h_{n}^{(\alpha, \beta)}$, the square of the norm of $P_{n}^{(\alpha, \beta)}$, is given by

$$
h_{n}^{(\alpha, \beta)}=\frac{(\alpha+1)_{n}(\beta+1)_{n}(\alpha+\beta+n+1)}{n !(\alpha+\beta+2)_{n}(\alpha+\beta+2 n+1)} .
$$

The Gegenbauer polynomials, denoted by $C_{n}^{\lambda}$, satisfy the orthogonal relation

$$
\left.c_{\lambda} \int_{-1}^{1} C_{n}^{\lambda}(t) C_{m}^{\lambda}(t)\left(1-t^{2}\right)^{\lambda-\frac{1}{2}} \mathrm{~d} t=\frac{\lambda}{n+\lambda} C_{n}^{(} 1\right) \delta_{n, m}, \quad \lambda>\frac{1}{2},
$$


and normalized by $C_{n}^{\lambda}(1)=(2 \lambda)_{n} / n$ !, where the constant $c_{\lambda}$ is as in (2.8). When $\lambda=0$, the Gegenbauer polynomial reduces to the Chebyshev polynomial $T_{n}$ of the first kind, defined by $T_{n}(\cos \theta)=\cos n \theta$. For convenience, we define

$$
Z_{n}^{\lambda}(t):=\frac{n+\lambda}{\lambda} C_{n}^{\lambda}(t), \quad \lambda>0, \quad \text { and } \quad Z_{n}^{0}(t):=\left\{\begin{array}{ll}
2 T_{n}(t) & n \geq 1 \\
1 & n=0
\end{array},\right.
$$

which will be used multiple times in our discussion below.

2.3. Orthogonal polynomials on the unit sphere and the unit ball. For $\kappa=$ $\left(\kappa_{1}, \ldots, \kappa_{d}\right) \in \mathbb{R}^{d}$, we define the weight function

$$
h_{\kappa}(x)=\prod_{k=0}^{d}\left|x_{i}\right|^{\kappa_{i}}, \quad \kappa_{i} \geq 0, \quad x \in \mathbb{R}^{d},
$$

which is invariant under the group $\mathbb{Z}_{2}^{d}$ of sign changes. On the unit sphere $\mathbb{S}^{d-1}$ of $\mathbb{R}^{d}$, we consider orthogonal polynomials with respect to the inner product

$$
\langle f, g\rangle_{\mathbb{S}}=c_{\kappa}^{h} \int_{\mathbb{S}^{d-1}} f(\xi) g(\xi) h_{\kappa}^{2}(\xi) \mathrm{d} \sigma_{\mathbb{S}}(\xi), \quad c_{\kappa}^{h}:=\frac{\Gamma\left(|\kappa|+\frac{d}{2}\right)}{2 \Gamma\left(\kappa_{1}+\frac{1}{2}\right) \cdots \Gamma\left(\kappa_{d}+\frac{1}{2}\right)},
$$

where $\mathrm{d} \sigma_{\mathbb{S}}$ denotes the surface measure of $\mathbb{S}^{d-1},|\kappa|=\kappa_{1}+\cdots+\kappa_{d}$, and it is normalized so that $\langle 1,1\rangle_{\mathbb{S}}=1$. The orthogonal polynomials for this inner product are a special case of Dunkl's $h$-harmonics for weight functions invariant under reflection groups. Let $\mathcal{H}_{n}\left(h_{\kappa}^{2}\right)$ be the of $h$-harmonics of degree $n$ in $d$ variables. It consists of homogeneous polynomials $Y$ of degree $n$ in $d$-variables that satisfy $\Delta_{h} Y=0$, where $\Delta_{h}$ is the Dunkl Laplacian, a second order differential-difference operator [5], which we shall not specify. If $Y \in \mathcal{H}_{n}^{d}\left(h_{\kappa}^{2}\right)$, then $Y(x)=r^{n} Y(\xi), x=r \xi$ with $\xi \in \mathbb{S}^{d-1}$. We shall also use $\mathcal{H}_{n}^{d}\left(h_{\kappa}^{2}\right)$ to denote the space of $Y(\xi)$, the restriction of $h$-harmonics on the unit sphere or spherical $h$-harmonics. When $\kappa=0$, or $h_{\kappa}(x)=1, \Delta_{h}$ becomes the ordinary Laplace operator $\Delta$ and the space $\mathcal{H}_{n}^{d}\left(h_{0}^{2}\right)$ becomes the space $\mathcal{H}_{n}^{d}$ of ordinary spherical harmonics of degree $n$ in $d$ variables. It is known that

$$
a_{n}^{d}:=\operatorname{dim} \mathcal{H}_{n}^{d}=\left(\begin{array}{c}
n+d-1 \\
n
\end{array}\right)-\left(\begin{array}{c}
n+d-3 \\
n-2
\end{array}\right) .
$$

The spherical $h$-harmonics are orthogonal polynomials in the sense that $\left\langle Y_{n}, Y_{m}\right\rangle_{\mathbb{S}}=$ 0 if $Y_{n}$ and $Y_{m}$ are spherical $h$-harmonics of degree $n$ and $m$ with $n \neq m$. An explicit orthogonal basis of $\mathcal{H}_{n}^{d}\left(h_{k}^{2}\right)$ can be given in terms of the Jacobi polynomials (cf. [6, p. 229]). The reproducing kernel of $\mathcal{H}_{n}^{d}\left(h_{\kappa}^{2}\right)$ satisfies a closed form formula, which is a generalization of the addition formula for the ordinary spherical harmonics. For $n=0,1,2, \ldots$, let $\mathrm{P}_{n}\left(h_{\kappa}^{2} ; \cdot, \cdot\right)$ be the reproducing kernel of $\mathcal{H}_{n}^{d}\left(h_{\kappa}^{2}\right)$. Then 20]

$$
\mathrm{P}_{n}\left(h_{\kappa}^{2} ; \xi, \eta\right)=V_{\kappa}\left[Z_{n}^{|\kappa|+\frac{d-2}{2}}\langle\xi, \cdot\rangle\right](\eta), \quad \xi, \eta \in \mathbb{S}^{d-1},
$$

where $V_{\kappa}$ is the integral operator, called intertwining operator, defined by

$$
V_{\kappa} f(x)=c_{\kappa-\frac{1}{2}} \int_{[1,1]^{d}} f\left(x_{1} u_{1}, \cdots, x_{d} u_{d}\right) \Phi_{\kappa}(u) \mathrm{d} u,
$$

where $c_{\kappa-\frac{1}{2}}=c_{\kappa_{1}-\frac{1}{2}} \cdots c_{k_{d}-\frac{1}{2}}$ with $c_{\lambda}$ as defined in (2.8) and

$$
\Phi_{\kappa}(u)=\prod_{i=1}^{d}\left(1+u_{i}\right)\left(1-u_{i}^{2}\right)^{\kappa_{i}-1} .
$$


If $\kappa_{i}=0$ for some $\kappa_{i}$, then the identity (2.14) is defined under the limit (2.9). In particular, if $\kappa_{i}=0$ for all $i$, then $h_{\kappa}(x)=1$ and the right-hand side of (2.13) reduces to $Z_{n}^{\frac{d-2}{2}}(\langle x, y\rangle)$, in which case (2.13) degenerates to the addition formula of the ordinary spherical harmonics. We need the following lemma proved in [20].

Lemma 2.2. Let $g: \mathbb{R} \mapsto \mathbb{R}$ be a function such that both integrals below are defined. Let $\alpha_{\kappa}=|k|+\frac{d-2}{2}$. Then, for $x \in \mathbb{R}^{d}$,

$$
c_{\kappa}^{h} \int_{\mathbb{S}^{d-1}} V_{\kappa}[f(\langle x, \cdot\rangle)](\eta) h_{\kappa}^{2}(\eta) d \sigma_{\mathbb{S}}(\eta)=c_{\alpha_{\kappa}} \int_{-1}^{1} f(\|x\| v)\left(1-v^{2}\right)^{\alpha_{\kappa}-\frac{1}{2}} \mathrm{~d} v .
$$

The closed formula (2.13) has been instrumental for recent progress on analysis on the sphere equipped with $h_{\kappa}^{2}$ weight; see, for example, [4, 6].

2.4. Orthogonal polynomials on the unit ball. It is known [6, Section 4.4] that orthogonal polynomials on the unit ball is closely related to orthogonal polynomials on the unit sphere. Let $h_{\kappa}$ be defined in (2.12). For the unit ball $\mathbb{B}^{d}$ of $\mathbb{R}^{d}$, we let

$$
\varpi_{\kappa, \mu}(x)=h_{\kappa}^{2}(x)\left(1-\|x\|^{2}\right)^{\mu-\frac{1}{2}}, \quad \mu>-\frac{1}{2}, \quad x \in \mathbb{B}^{d},
$$

and consider orthogonal polynomials with respect to the inner product

$$
\langle f, g\rangle_{\mathbb{B}}=b_{\kappa, \mu} \int_{\mathbb{B}^{d}} f(x) g(x) \varpi_{\kappa, \mu}(x) \mathrm{d} x,
$$

where $b_{\kappa, \mu}$ is a normalization constant such that $\langle 1,1\rangle_{\mathbb{B}}=1$. For $\kappa \in \mathbb{R}^{d}$, we define $\kappa_{d+1}=\mu$ and $\boldsymbol{\kappa}=\left(\kappa, \kappa_{d+1}\right) \in \mathbb{R}^{d+1}$. For $x \in \mathbb{B}^{d}$, we define $x_{d+1}=\sqrt{1-\|x\|^{2}}$ and $X=\left(x, x_{d+1}\right)$ as well as $X^{-}=\left(x, x_{d+1}\right)$; then $X \in \mathbb{S}^{d}$ and $X^{-} \in \mathbb{S}^{d}$. It is easy to verify that the measure $\varpi_{\kappa, \mu} \mathrm{d} x$ on $\mathbb{B}^{d}$ satisfies

$$
\varpi_{\kappa, \mu}(x) \mathrm{d} x=\prod_{i=1}^{d+1}\left|x_{i}\right|^{2 \kappa_{i}} d \sigma(X)=h_{\kappa}^{2}(X) \mathrm{d} \sigma_{\mathbb{S}^{d}}(X),
$$

where $\mathrm{d} \sigma_{\mathbb{S}^{d}}$ is the surface measure of $\mathbb{S}^{d}$. By symmetry, it follows that

$$
\int_{\mathbb{B}^{d}} f(x) \varpi_{\kappa, \mu}(x) \mathrm{d} x=\frac{1}{2} \int_{\mathbb{S}^{d}}\left[f(X)+f\left(X^{-}\right)\right] h_{\kappa}^{2}(X) \mathrm{d} \sigma_{\mathbb{S}^{d}}(X) .
$$

Let $\mathcal{V}_{n}\left(\mathbb{B}^{d}, \varpi_{\kappa, \mu}\right)$ be the space of orthogonal polynomials with respect to the inner product $\langle\cdot, \cdot\rangle_{\mathbb{B}}$. We then have the decomposition

$$
\mathcal{H}_{n}^{d+1}\left(h_{\kappa}\right)=\mathcal{V}_{n}\left(\mathbb{B}^{d}, \varpi_{\kappa, \mu}\right) \bigoplus x_{d+1} \mathcal{V}_{n-1}\left(\mathbb{B}^{d}, \varpi_{\kappa, \mu+1}\right)
$$

In particular, a basis for $\mathcal{V}_{n}\left(\mathbb{B}^{d}, \varpi_{\kappa, \mu}\right)$ can be derived from those spherical $h$-harmonics in $\mathcal{H}_{n}^{d+1}\left(h_{\kappa}\right)$ that are even in the $x_{d+1}$ variable. Moreover, we can also derive a close formula for the reproducing kernel of $\mathcal{V}_{n}\left(\mathbb{B}^{d}, \varpi_{\kappa, \mu}\right)$ through this correspondence.

\section{LAGUERRE EXPANSIONS ON THE CONIC SURFACE}

We work on the conic surface that is standardized by

$$
\mathbb{V}_{0}^{d+1}=\left\{(x, t) \in \mathbb{R}^{d+1}:\|x\|=t, x \in \mathbb{R}^{d}, t \in \mathbb{R}_{+}\right\}, \quad d \geq 2 .
$$

For $\kappa \geq 0$, we define the Laguerre type weight function $\mathrm{w}_{\kappa}$ on the conic surface by

$$
\mathrm{w}_{\kappa}(x, t)=h_{\kappa}^{2}(x) t^{-1} \mathrm{e}^{-t}, \quad(x, t) \in \mathbb{V}_{0}^{d+1},
$$


where $h_{\kappa}$ is given in (2.12). In the first subsection, we discuss orthogonal polynomials with respect to $w_{\kappa}$. In the second subsection we derive closed form formulas for the Poisson kernel and the reproducing kernels of these polynomials.

3.1. Orthogonal polynomials. For $d \geq 2$, we define the inner product with respect to $\mathrm{w}_{\kappa}$ on the polynomial space $\mathbb{R}[x, t] /\left\langle\|x\|^{2}-t^{2}\right\rangle$ by

$$
\langle f, g\rangle_{\kappa}=\mathrm{b}_{\kappa} \int_{\mathbb{V}_{0}^{d+1}} f(x, t) g(x, t) \mathrm{w}_{\kappa}(x, t) \mathrm{d} \sigma(x, t),
$$

where $\mathrm{d} \sigma(x, t)$ denotes the Lebesgue measure on the conic surface and $\mathrm{b}_{\kappa}$ is the normalization constant chosen so that $\langle 1,1\rangle_{\kappa}=1$. The value of $b_{\kappa}$ is given by

$$
\mathrm{b}_{\kappa}=\frac{1}{\int_{\mathbb{V}_{0}^{d+1}} \mathrm{w}_{\kappa}(x, t) \mathrm{d} \sigma(x, t)}=\frac{c_{\kappa}^{h}}{\Gamma(2|\kappa|+d-1)}=b_{2|\kappa|+d-2} c_{\kappa}^{h},
$$

evaluated by writing the integral over $\mathbb{V}_{0}^{d+1}$ as, using the spherical-polar coordinates,

$$
\int_{\mathbb{V}_{0}^{d+1}} f(x, t) d \sigma(x, t)=\int_{0}^{\infty} t^{d-1} \int_{\mathbb{S}^{d-1}} f(t \xi, t) \mathrm{d} \sigma_{\mathbb{S}}(\xi) \mathrm{d} t .
$$

Let $\mathcal{V}_{n}\left(\mathbb{V}_{0}^{d+1}, \mathrm{w}_{\kappa}\right)$ denote the space of orthogonal polynomials of degree $n$ with respect to this inner product. The space has the same dimension as that of $\mathcal{H}_{n}^{d+1}$, so that

$$
\operatorname{dim} \mathcal{V}_{n}\left(\mathbb{V}_{0}^{d+1}, \mathrm{w}_{\kappa}\right)=\left(\begin{array}{c}
n+d \\
n
\end{array}\right)-\left(\begin{array}{c}
n+d-2 \\
n-2
\end{array}\right)
$$

Using (3.1), we see that a family of orthogonal polynomials on the conic surface can be given in terms of $h$-spherical harmonics and the Laguerre polynomials.

Proposition 3.1. Let $\left\{Y_{\ell}^{m}: 1 \leq \ell \leq a_{m}^{d}\right\}$ be an orthonormal basis of $\mathcal{H}_{m}^{d}\left(h_{\kappa}^{2}\right)$. Define

$$
\mathrm{L}_{m, \ell}^{n}(x, t)=L_{n-m}^{2 m+2|\kappa|+d-2}(t) Y_{\ell}^{m}(x), \quad 0 \leq m \leq n, \quad 1 \leq \ell \leq a_{m}^{d} .
$$

Then $\left\{\mathrm{L}_{m, \ell}^{n}: 0 \leq m \leq n, \quad 1 \leq \ell \leq a_{m}^{d}\right\}$ is an orthogonal basis of $\mathcal{V}_{n}\left(\mathbb{V}_{0}^{d+1}, \mathrm{w}_{\kappa}\right)$. Moreover, the norm square of $\mathrm{L}_{m, \ell}^{n}$ is given by

$$
\mathrm{h}_{m, n}:=\left\langle\mathrm{L}_{m, \ell}^{n}, \mathrm{~L}_{m, \ell}^{n}\right\rangle_{\kappa}=\frac{(2|\kappa|+d-1)_{n+m}}{(n-m) !} .
$$

Proof. Let $\alpha=|\kappa|+\frac{d-2}{2}$. Then, using (3.1), we obatin

$$
\begin{aligned}
\left\langle\mathrm{L}_{m, \ell}^{n}, \mathrm{~L}_{m^{\prime}, \ell^{\prime}}^{n^{\prime}}\right\rangle_{\kappa,-1}= & b_{2 \alpha} \int_{0}^{\infty} L_{n-m}^{2 m+\alpha}(t) L_{n^{\prime}-m^{\prime}}^{2 m+\alpha}(t) t^{2 m+2 \alpha} e^{-t} \mathrm{~d} t \\
& \times c_{\kappa}^{h} \int_{\mathbb{S}^{d}-1} Y_{\ell}^{m}(\xi) Y_{\ell^{\prime}}^{m^{\prime}}(\xi) h_{\kappa}^{2}(\xi) \mathrm{d} \sigma_{\mathbb{S}}(\xi) \\
= & \frac{\Gamma(2 m+2 \alpha+1)}{\Gamma(2 \alpha+1)} h_{n-m}^{2 m+2 \alpha} \delta_{n, n^{\prime}} \delta_{m, m^{\prime}} \delta_{\ell, \ell^{\prime \prime}},
\end{aligned}
$$

where $h_{n-m}^{\alpha}$ is the norm square of the Laguerre polynomial $L_{n-m}^{\alpha}$.

We could consider orthogonal polynomials with respect to the weight function $h_{\kappa}^{2}(x) t^{\beta} \mathrm{e}^{-t}$ for $\beta \neq-1$. However, the case $\beta=-1$ appears to be the most natural setting for several reasons 22 . In particular, the closed form formula of the reproducing kernels is of a relative simple form for $\beta=-1$; see Theorem 3.7 below. 
We call the polynomials in (3.2) Laguerre type polynomials when $\kappa \neq 0$, and Laguerre polynomials when $\kappa=0$, on the conic surface. For $f \in L^{2}\left(\mathbb{V}_{0}^{d+1}, \mathbf{w}_{\kappa}\right)$, the Fourier-Laguerre expansion of $f$ is defined by

$$
f=\sum_{n=0}^{\infty} \sum_{m=0}^{n} \sum_{\ell=1}^{a_{m}^{d}} \widehat{f}_{m, \ell}^{n} L_{m, \ell}^{n} \quad \text { with } \quad \widehat{f}_{m, \ell}^{n}=\frac{\left\langle f, \mathrm{~L}_{m, \ell}^{n}\right\rangle_{\kappa}}{\mathrm{h}_{m, n}}
$$

The projection operator $\operatorname{proj}_{n}\left(\mathrm{w}_{\kappa}\right): L^{2}\left(\mathbb{V}_{0}^{d+1}, \mathrm{w}_{\kappa}\right) \mapsto \mathcal{V}_{n}\left(\mathbb{V}_{0}^{d+1}, \mathrm{w}_{\kappa}\right)$ and the $n$-th partial sum operator $S_{n}\left(w_{\kappa}\right)$ of this expansion are defined by

$$
\operatorname{proj}_{n}\left(\mathrm{w}_{\kappa} ; f\right)=\sum_{m=0}^{n} \sum_{\ell=1}^{a_{m}^{d}} \widehat{f}_{m, \ell}^{n} \mathrm{~L}_{m, \ell}^{n} \quad \text { and } \quad \mathrm{S}_{n} f\left(\mathrm{w}_{\kappa} ; f\right)=\sum_{m=0}^{n} \operatorname{proj}_{m} f\left(\mathrm{w}_{\kappa} ; f\right) \text {. }
$$

If $f(x, t)$ depends only on $t$, then $\widehat{f}_{m, \ell}^{n}=0$ for all $m>0$ and $\widehat{f}_{0, \ell}^{n}$ reduces to the classical Fourier-Laguerre coefficients of one variable. In particular, the following proposition follows readily.

Proposition 3.2. Let $f(x, t)=f_{0}(t)$ and $f_{0} \in L^{2}\left(\mathbb{R}_{+} ; w_{2|\kappa|+d-2}\right)$. Then the FourierLaguerre expansion of $f$ on the conic surface becomes the Laguerre expansion of $f_{0}$ in $L^{2}\left(\mathbb{R}_{+} ; w_{2|\kappa|+d-2}\right)$. In particular,

$$
\mathrm{S}_{n}\left(\mathrm{w}_{\kappa} ; f,(x, t)\right)=s_{n}\left(w_{2|\kappa|+d-2} ; f_{0}, t\right), \quad n=0,1,2, \ldots
$$

The projection operator $\operatorname{proj}_{n}\left(w_{\kappa}\right)$ of the Fourier-Laguerre series can be written as an integral operator

$$
\operatorname{proj}_{n}\left(\mathrm{w}_{\kappa}\right) f=\mathrm{b}_{\kappa} \int_{\mathbb{V}_{0}^{d+1}} f(y, s) \mathrm{P}_{n}\left(\mathrm{w}_{\kappa} ;(x, t),(y, s)\right) \mathrm{w}_{\kappa}(y, s) \mathrm{d} \sigma(y, s),
$$

where $\mathrm{P}_{n}\left(\mathrm{w}_{\kappa} ; \cdot, \cdot\right)$ is the reproducing kernel of the space $\mathcal{V}_{n}\left(\mathbb{V}_{0}^{d+1}, \mathrm{w}_{\kappa}\right)$, which can be written in terms of the orthogonal basis $\mathrm{L}_{m, \ell}^{n}$ as

$$
\mathrm{P}_{n}\left(\mathrm{w}_{\kappa} ;(x, t),(y, s)\right)=\sum_{m=0}^{n} \sum_{\ell=1}^{a_{m}^{d}} \frac{\mathrm{L}_{m, \ell}^{n}(x, t) \mathrm{L}_{m, \ell}^{n}(y, s)}{\mathrm{h}_{m, n}} .
$$

The Laguerre type polynomials on the conic surface can be derived from the Jacobi type polynomials on the conic surface via a limit process. The latter polynomials are orthogonal with respect to the inner product $\langle\cdot, \cdot\rangle_{w_{\kappa, \gamma}}$ defined by

$$
\langle f, g\rangle_{\kappa, \gamma}=\mathrm{b}_{\kappa, \gamma} \int_{\mathbb{V}_{0}^{d+1}} f(x, t) g(x, t) \mathrm{w}_{\kappa, \gamma}(x, t) h_{\kappa}^{2}(x, t) \mathrm{d} \sigma(x, t),
$$

where $\mathrm{b}_{\kappa, \gamma}=c_{\kappa}^{h} c_{2|\kappa|+d-1, \gamma}$, with $c_{\alpha, \beta}$ defined in (2.10), and the weight function $\mathrm{w}_{\kappa, \gamma}$ is given by

$$
\mathrm{w}_{\kappa, \gamma}(t)=h_{\kappa}^{2}(x) t^{-1}(1-t)_{+}^{\gamma}, \quad \kappa_{i} \geq 0, \quad \gamma>-1,
$$

where $(1-t)_{+}^{\gamma}=(1-t)^{\gamma}$ if $t<1$ and 0 if $t \geq 0$. Let $\mathcal{V}_{n}\left(\mathbb{V}_{0}^{d+1}, \mathrm{w}_{\kappa, \gamma}\right)$ be the corresponding space of orthogonal polynomials of degree $n$. An orthogonal basis of this space can be given by the Jacobi polynomials and spherical $h$-harmonics [22].

Proposition 3.3. Let $\left\{Y_{\ell}^{m}: 1 \leq \ell \leq a_{m}^{d}\right\}$ be an orthonormal basis of $\mathcal{H}_{m}^{d}\left(h_{\kappa}^{2}\right)$. Define

$$
\mathrm{J}_{m, \ell}^{n}(x, t)=P_{n-m}^{(2 m+2|\kappa|+d-2, \gamma)}(1-2 t) Y_{\ell}^{m}(x), \quad 1 \leq \ell \leq a_{m}^{d}, 0 \leq m \leq n .
$$


Then $\left\{\mathrm{J}_{m, \ell}^{n}: 0 \leq m \leq n, \quad 1 \leq \ell \leq a_{m}^{d}\right\}$ is an orthogonal basis of $\mathcal{V}_{n}\left(\mathbb{V}_{0}^{d+1}, \mathrm{w}_{\kappa, \gamma}\right)$. Moreover, the norm square of $\mathrm{J}_{m, \ell}^{n}$ is given by

$$
\mathrm{h}_{m, n}^{\mathrm{J}}=\left\langle\mathrm{J}_{m, \ell}^{n}, \mathrm{~J}_{m, \ell}^{n}\right\rangle_{\mathrm{w}_{\kappa, \gamma}}=\frac{(2|\kappa|+d-1)_{2 m}}{(2|\kappa|+\gamma+d)_{2 m}} h_{n-m}^{(2 m+2|\kappa|+d-2, \gamma)},
$$

where $h_{n}^{(\alpha, b)}$ is the norm square of the Jacobi polynomial of degree $n$.

The proof follows exactly as that of Proposition 3.1 using the Jacobi polynomials. We denote the reproducing kernel of $\mathcal{V}_{n}\left(\mathbb{V}_{0}^{d+1}, \mathrm{w}_{\kappa, \gamma}\right)$ by $\mathrm{P}_{n}^{J}\left(\mathrm{w}_{\kappa, \gamma} ; \cdot, \cdot\right)$. Then

$$
\mathrm{P}_{n}^{\mathrm{J}}\left(\mathrm{w}_{\kappa, \gamma} ;(x, t),(y, s)\right)=\sum_{m=0}^{n} \sum_{\ell=1}^{a_{m}^{d}} \frac{\mathrm{J}_{m, \ell}^{n}(x, t) \mathrm{J}_{m, \ell}^{n}(y, s)}{\mathrm{h}_{m, n}^{J}} .
$$

Proposition 3.4. For $d \geq 2$,

$$
\lim _{\gamma \rightarrow \infty} \gamma^{m} \mathrm{~J}_{m, \ell}^{n}\left(\frac{x}{\gamma}, \frac{t}{\gamma}\right)=\mathrm{L}_{m, \ell}^{n}(x, t) \quad \text { and } \quad \lim _{\gamma \rightarrow \infty} \gamma^{2 m} \mathrm{~h}_{m, n}^{\mathrm{J}}=\mathrm{h}_{m, n} .
$$

Furthermore, for the reproducing kernels,

$$
\lim _{\gamma \rightarrow \infty} \mathrm{P}_{n}^{J}\left(\mathrm{w}_{\kappa, \gamma} ;\left(\frac{x}{\gamma}, \frac{t}{\gamma}\right),\left(\frac{y}{\gamma}, \frac{s}{\gamma}\right)\right)=\mathrm{P}_{n}\left(\mathrm{w}_{\kappa} ;(x, t),(y, s)\right) .
$$

Proof. Since the Laguerre polynomials are limits of the Jacobi polynomials [16, (5.3.4)],

$$
\lim _{\beta \rightarrow \infty} P_{n}^{(\alpha, \beta)}\left(1-2 \beta^{-1} t\right)=L_{n}^{\alpha}(t),
$$

the limits in (3.4) follow accordingly using $Y_{\ell}^{m}\left(\gamma^{-1} x\right)=\gamma^{-m} Y_{\ell}^{m}(x)$ and the explicit formula of $h_{n}^{(\alpha, \beta)}$. The limit (3.5) follows as a consequence using the expression of both kernels written in terms of their corresponding orthogonal bases.

The reproducing kernel of the Jacobi polynomials on the conic surface satisfies a closed form formula, discovered recently in [22, given via $Z_{n}^{\lambda}$ in (2.11).

Theorem 3.5. Let $d \geq 2, \kappa_{i} \geq 0$ and $\gamma \geq-\frac{1}{2}$. Let $\alpha_{\kappa}=|\kappa|+\frac{d-2}{2}$. Then, for $(x, t),(y, s) \in \mathbb{V}_{0}^{d+1}$,

$$
\begin{aligned}
\mathrm{P}_{n}^{\mathrm{J}}\left(\mathrm{w}_{\kappa, \gamma} ;(x, t),(y, s)\right)= & c_{\kappa, \gamma} \int_{[-1,1]^{d+2}} Z_{2 n}^{2 \alpha_{\kappa}+\gamma+1}(\zeta(x, t, y, s ; u, v)) \\
& \times\left(1-v_{1}^{2}\right)^{\alpha_{\kappa}-1}\left(1-v_{2}^{2}\right)^{\gamma-\frac{1}{2}} \mathrm{~d} v \Phi_{\kappa}(u) \mathrm{d} u
\end{aligned}
$$

where $c_{\kappa, \gamma}=c_{\gamma} c_{\alpha-\frac{1}{2}} c_{\kappa-\frac{1}{2}}$ and $\zeta(x, t, y, s ; v)=v_{1} \rho(x, t, y, s ; u)+v_{2} \sqrt{1-t} \sqrt{1-s}$ with

$$
\rho(x, t, y, s ; u):=\sqrt{\frac{1}{2}\left(t s+x_{1} y_{1} u_{1}+\cdots+x_{d} y_{d} u_{d}\right)} ;
$$

moreover, the identity holds under the limit (2.9) if either $\gamma=-\frac{1}{2}$, or $d=2$, or $\kappa_{i}=0$ for one or more $i$.

The formula (3.6) is stated in [22, Theorem 10.2] where, however, $|\kappa|$ should be replaced by $2|\kappa|$. There is also a more general formula when the weight function is $h_{\kappa}^{2}(x) t^{\beta}(1-t)^{\gamma}$, which however is more involved for our purpose. 
3.2. Poisson kernel and reproducing kernels. Our main results in this subsection are the closed form formulas for the Poisson kernels and the reproducing kernels of orthogonal polynomials on the conic surface. We start with the Poisson kernels $\mathrm{P}\left(\mathrm{w}_{\kappa} ; r, \cdot, \cdot\right)$ defined by

$$
\mathrm{Q}_{r}\left(\mathrm{w}_{\kappa} ;(x, t),(y, s)\right)=\sum_{n=0}^{\infty} \mathrm{P}_{n}\left(\mathrm{w}_{\kappa} ; x, t, y, s\right) r^{n}, \quad 0 \leq r<1 .
$$

Theorem 3.6. Let $d \geq 2, \kappa_{i} \geq 0$. If $\alpha_{\kappa}=|\kappa|+\frac{d-2}{2}>0$, then

$$
\begin{aligned}
\mathrm{Q}_{r}\left(\mathrm{w}_{\kappa} ;(x, t),(y, s)\right)= & \frac{\mathrm{e}^{-\frac{(t+s) r}{1-r}}}{(1-r)^{2 \alpha_{\kappa}+1}} c_{\alpha_{\kappa}-\frac{1}{2}} c_{\kappa-\frac{1}{2}} \int_{[-1,1]^{d+1}} \exp \left\{\frac{2 \sqrt{r} v}{1-r} \rho(x, t, y, s ; u)\right\} \\
& \times \Phi_{\kappa}(u) \mathrm{d} u\left(1-v^{2}\right)^{\alpha_{\kappa}-1} \mathrm{~d} v,
\end{aligned}
$$

where $\Phi_{\kappa}$ is in (2.15); moreover, if $\alpha_{\kappa}=0$ or, equivalently, $\kappa=0$ and $d=2$, then

$$
\mathrm{Q}_{r}\left(\mathrm{w}_{0} ;(x, t),(y, s)\right)=\frac{\mathrm{e}^{-\frac{(t+s) r}{1-r}}}{1-r} \cosh \left(\frac{2 \sqrt{r}}{1-r} \rho(x, t, y, s)\right)
$$

where $\rho(x, t, y, s)=\rho(x, t, y, s ; 1)=\sqrt{\frac{1}{2}(t s+\langle x, y\rangle)}$.

Proof. We start with (3.6) and change variable $v_{2} \mapsto r=\zeta\left(x, t, y, s ; u, v_{1}, v_{2}\right)$ in the integral against $v_{2}$, so that

$$
v_{2}=\frac{r-v_{1} \rho(x, t, y, s ; u)}{\sqrt{1-t} \sqrt{1-s}} \quad \text { and } \quad \mathrm{d} v_{2}=\frac{1}{\sqrt{1-t} \sqrt{1-s}} \mathrm{~d} r .
$$

This leads to a reformulation of $\mathrm{P}_{n}^{J}\left(\mathrm{w}_{\kappa, \gamma} ; \cot , \cdot\right)$ as

$$
\mathrm{P}_{n}^{J}\left(\mathrm{w}_{\kappa, \gamma} ;(x, t),(y, s)\right)=c_{\gamma} \int_{-1}^{1} Z_{2 n}^{\lambda}(r) G_{\gamma}(x, t, y, s ; u ; r)\left(1-r^{2}\right)^{\lambda-\frac{1}{2}} \mathrm{~d} r,
$$

where $\lambda=\gamma+2 \alpha_{\kappa}+1=\gamma+2|\kappa|+d-1$ and

$$
G_{\gamma}(x, t, y, s ; u ; r)=c_{\alpha_{\kappa}-\frac{1}{2}} c_{\kappa-\frac{1}{2}} \int_{[-1,1]^{d+1}} F_{\gamma}(x, t, y, s, r ; u, v)\left(1-v^{2}\right)^{\alpha_{\kappa}-1} \mathrm{~d} v \Phi_{\kappa}(u) \mathrm{d} u
$$

in terms of the the function $F_{\gamma}$ defined by

$$
F_{\gamma}(x, t, y, s, r ; u, v)=\frac{1}{\sqrt{1-t} \sqrt{1-s}\left(1-r^{2}\right)^{\lambda-\frac{1}{2}}}\left(1-\frac{(r-v \rho(x, t, y, s ; u))^{2}}{(1-t)(1-s)}\right)^{\gamma-\frac{1}{2}}
$$

if $|r-v \rho(x, t, y, s ; u)| \leq \sqrt{1-t} \sqrt{1-s}$ and $F_{\gamma}(x, t, y, s, r ; u, v)=0$ otherwise. Let us define

$$
\widetilde{G}_{\gamma}(x, t, y, s ; u, r)=\frac{1}{2}\left[G_{\gamma}(x, t, y, s ; u, r)+G_{\gamma}(x, t, y, s ; u,-r)\right],
$$

which is even in $r$. By symmetry of the integral, it follows from (3.9) that

$c_{\gamma} \int_{-1}^{1} Z_{m}^{\lambda}(r) \widetilde{G}_{\gamma}(x, t, y, s ; u, r)\left(1-r^{2}\right)^{\lambda-\frac{1}{2}} \mathrm{~d} r= \begin{cases}\mathrm{P}_{n}^{J}\left(w_{-1, \gamma} ;(x, t),(y, s)\right), & m=2 n, \\ 0, & m=2 n+1 .\end{cases}$

The integral in the left-hand side gives the Fourier coefficient, up to the normalization constant, of the function $r \mapsto \widetilde{G}_{\gamma}(x, t, y, s ; r)$ of the Fourier-Gegenbauer series in 
$L^{2}\left([-1,1],\left(1-\{\cdot\}^{2}\right)^{\lambda-\frac{1}{2}}\right)$. Consequently, using $h_{n}^{\lambda}=\frac{n+\lambda}{\lambda} C_{n}^{\lambda}(1)$, it follows that

$$
\widetilde{G}_{\gamma}(x, t, y, s ; r)=\frac{c_{\gamma}}{c_{\lambda}} \sum_{n=0}^{\infty} \mathrm{P}_{n}^{J}\left(\mathrm{w}_{\kappa, \gamma} ;(x, t),(y, s)\right) \frac{C_{2 n}^{\lambda}(r)}{C_{2 n}^{\lambda}(1)}
$$

Since $\lambda=\gamma+2 \alpha_{\kappa}+1, c_{\gamma} / c_{\lambda} \rightarrow 1$ as $\gamma \rightarrow \infty$ and, by the ${ }_{2} F_{1}$ expression of $C_{n}^{\lambda}$, it is easy to see that $C_{2 n}^{\lambda}(r) / C_{2 n}^{\lambda}(1) \mapsto r^{2 n}$ as $\gamma \rightarrow \infty$, it follows from (3.4) that

$$
\lim _{\gamma \rightarrow \infty} \widetilde{G}_{\gamma}(x, t, y, s ; r)=\mathrm{Q}_{r^{2}}\left(\mathrm{w}_{\kappa} ;(x, t),(y, s)\right)
$$

Since $\rho(x / \gamma, t \gamma, y \gamma, s \gamma ; u)=\frac{1}{\gamma} \rho(x, t, y, s ; u)$, we see that $F_{\gamma}$ satisfies

$$
\begin{aligned}
F_{\gamma}\left(\frac{x}{\gamma}, \frac{t}{\gamma}, \frac{y}{\gamma}, \frac{s}{\gamma}, r ; u, v\right) & =\frac{1}{\left(1-r^{2}\right)^{2 \alpha_{\kappa}+1}\left(1-\frac{t}{\gamma}\right)^{\gamma}\left(1-\frac{s}{\gamma}\right)^{\gamma}} \\
& \times\left(1-\frac{t+s-2 v \rho(x, t, y, s ; u)}{\gamma\left(1-r^{2}\right)}-\frac{t s+v^{2} \rho(x, t, y, s ; u)^{2}}{\gamma^{2}\left(1-r^{2}\right)}\right)^{\gamma-\frac{1}{2}}
\end{aligned}
$$

if $r-\frac{v}{\gamma} \rho(x, t, y, s ; u) \leq \sqrt{1-\frac{t}{\gamma}} \sqrt{1-\frac{s}{\gamma}}$. Taking the limit $\gamma \rightarrow \infty$, we obtain

$$
\begin{aligned}
\lim _{\gamma \rightarrow \infty} F_{\gamma}\left(\frac{x}{\gamma}, \frac{t}{\gamma}, \frac{y}{\gamma}, \frac{s}{\gamma}, r ; u, v\right) & =\frac{1}{\left(1-r^{2}\right)^{2 \alpha_{\kappa}+1} \mathrm{e}^{-t-s}} \exp \left\{-\frac{t+s-2 v r \rho(x, t, y, s ; u)}{1-r^{2}}\right\} \\
& =\frac{1}{\left(1-r^{2}\right)^{2 \alpha_{\kappa}+1}} \exp \left\{-\frac{(t+s) r^{2}-2 v r \rho(x, t, y, s ; u)}{1-r^{2}}\right\}
\end{aligned}
$$

Hence, taking the limit of $G_{\gamma}$ accordingly, we conclude that

$$
\begin{aligned}
\lim _{\gamma \rightarrow \infty} G_{\gamma}\left(\frac{x}{\gamma}, \frac{t}{\gamma}, \frac{y}{\gamma}, \frac{s}{\gamma}, r ; v\right)= & \frac{1}{\left(1-r^{2}\right)^{2 \alpha_{\kappa}+1}} \mathrm{e}^{-\frac{(t+s) r^{2}}{1-r^{2}}} \int_{[-1,1] d+1} \exp \left\{\frac{2 r \rho(x, t, y, s ; u)}{1-r^{2}} v\right\} \\
& \times c_{\alpha-\frac{1}{2} c_{\alpha_{\kappa}-\frac{1}{2}}}\left(1-v^{2}\right)^{\alpha-1} \mathrm{~d} v \Phi_{\kappa}(u) \mathrm{d} u .
\end{aligned}
$$

Changing variable $v \rightarrow-v$ shows that the right-hand side is an even function in $r$. Hence, the limit of $\widetilde{G}_{\gamma}$ is the sam as the limit of $G_{\gamma}$. Thus, comparing the above limit with (3.10), we complete the proof.

The Poisson kernel can be regarded as the generating function of the reproducing kernels. We derive a closed formula of the latter from that of the former.

Theorem 3.7. Let $d \geq 2$. If $\alpha_{\kappa}=|\kappa|+\frac{d-2}{2}>0$, then for $(x, t),(y, s) \in \mathbb{V}_{0}^{d+1}$,

$$
\begin{aligned}
& \mathrm{P}_{n}\left(\mathrm{w}_{\kappa} ;(x, t),(y, s)\right)=C_{\kappa} \int_{[1,1]^{d}} \int_{0}^{\pi} L_{n}^{2 \alpha_{\kappa}}(t+s+2 \rho(x, t, y, s ; u) \cos \theta) \\
& \times e^{-\rho(x, t, y, s ; u) \cos \theta} j_{\alpha_{\kappa}-1}(\rho(x, t, y, s ; u) \sin \theta)(\sin \theta)^{2 \alpha_{\kappa}-1} \mathrm{~d} \theta \Phi_{\kappa}(u) \mathrm{d} u
\end{aligned}
$$


where $C_{\kappa}=\frac{2^{\alpha_{\kappa}-1} \Gamma\left(\alpha_{\kappa}+\frac{1}{2}\right)}{\sqrt{\pi}} c_{\kappa-\frac{1}{2}}$ and $\rho(\cdot)$ is given in (3.7). If $\alpha_{\kappa}=0$ or equivalently, $d=2$ and $|\kappa|=0$, then

$$
\begin{aligned}
\mathrm{P}_{n}\left(\mathrm{w}_{\kappa} ;(x, t),(y, s)\right)=\frac{1}{2}\left[\mathrm{e}^{-\rho(x, t, y, s)} L_{n}^{0}(t+s+2 \rho(x, t, y, s))\right. \\
\left.+\mathrm{e}^{\rho(x, t, y, s)} L_{n}^{0}(t+s-2 \rho(x, t, y, s))\right] \\
-\frac{1}{2} \rho(x, t, y, s)^{2} \int_{0}^{\pi} L_{n}^{0}(t+s+2 \rho(x, t, y, s) \cos \theta) \\
\quad \times e^{-\rho(x, t, y, s) \cos \theta} j_{1}(\rho(x, t, y, s) \sin \theta) \sin \theta \mathrm{d} \theta .
\end{aligned}
$$

In particular, for $d \geq 2$ and $\alpha_{\kappa} \geq 0$,

$$
\mathrm{P}_{n}\left(\mathrm{w}_{\kappa} ;(x, t),(0,0)\right)=L_{n}^{2|\kappa|+d-2}(t), \quad(x, t) \in \mathbb{V}_{0}^{d+1} .
$$

Proof. We can compare the Poisson formula for $\mathrm{Q}_{r}\left(\mathrm{w}_{\kappa} ; \cdot, \cdot\right)$ with (2.7) of the Laguerre polynomials by setting

$$
\begin{aligned}
& t^{*}=\frac{1}{2}\left(t+s-\sqrt{t^{2}+s^{2}-2\left(x_{1} y_{1} u_{1}+\cdots+x_{d} y_{d} u_{d}\right)}\right), \\
& s^{*}=\frac{1}{2}\left(t+s+\sqrt{t^{2}+s^{2}-2\left(x_{1} y_{1} u_{1}+\cdots+x_{d} y_{d} u_{d}\right)}\right),
\end{aligned}
$$

which are well defined, since the expression under the square root is nonnegative for $\|x\|=t$ and $\|y\|=s$ by the Cauchy inequality, and they satisfy

$$
t^{*}+s^{*}=t+s \quad \text { and } \quad \sqrt{t^{*} s^{*}}=\rho(x, t, y, s ; u) .
$$

Comparing the formula in Theorem 3.6 with (2.7), we obtain with $\alpha=\alpha_{\kappa}$,

$$
\mathrm{Q}_{r}\left(\mathrm{w}_{\kappa} ; x, t, y, s\right)=\frac{1}{(1-r)^{\alpha+\frac{1}{2}}} c_{\kappa-\frac{1}{2}} \int_{[-1,1]^{d}} \sum_{n=0}^{\infty} \frac{L_{n}^{\alpha-\frac{1}{2}}\left(t^{*}\right) L_{n}^{\alpha-\frac{1}{2}}\left(s^{*}\right)}{L_{n}^{\alpha-\frac{1}{2}}(0)} r^{n} \Phi_{\kappa}(u) \mathrm{d} u .
$$

If $\alpha>0$, we apply the product formula (2.3) on $L_{n}^{\alpha-\frac{1}{2}}\left(t^{*}\right) L_{n}^{\alpha-\frac{1}{2}}\left(s^{*}\right)$ which gives an integral representation that contains only $t^{*}+s^{*}$ and $t^{*} s^{*}$, so that we obtain, by (3.14),

$$
\begin{aligned}
\frac{L_{n}^{\alpha-\frac{1}{2}}\left(t^{*}\right) L_{n}^{\alpha-\frac{1}{2}}\left(s^{*}\right)}{L_{n}^{\alpha-\frac{1}{2}}(0)}= & \frac{2^{\alpha-\frac{1}{2}} \Gamma\left(\alpha+\frac{1}{2}\right)}{\sqrt{2 \pi}} \sum_{n=0}^{\infty} \int_{0}^{\pi} L_{n}^{\alpha-\frac{1}{2}}(t+s+2 \rho(x, t, y, s ; u) \cos \theta) \\
& \times e^{-\rho(x, t, y, s ; u) \cos \theta} j_{\alpha-1}(\rho(x, t, y, s ; u) \sin \theta)(\sin \theta)^{2 \alpha-1} \mathrm{~d} \theta .
\end{aligned}
$$

From the generating function (2.1) it follows readily that

$$
\frac{1}{(1-r)^{\beta+1}} \sum_{n=0}^{\infty} L_{n}^{\alpha}(x) r^{n}=\sum_{n=0}^{\infty} L_{n}^{\alpha+\beta+1}(x) r^{n} .
$$

Consequently, putting the last three displayed formula together, we deduce that

$$
\begin{aligned}
\mathrm{Q}_{r}\left(\mathrm{w}_{\kappa} ; x, t, y, s\right) & =C_{\kappa} \int_{[-1,1]^{d}} \int_{0}^{\pi} L_{n}^{2 \alpha}(t+s+2 \rho(x, t, y, s ; u) \cos \theta) r^{n} \\
& \times e^{-\rho(x, t, y, s ; u) \cos \theta} j_{\alpha-1}(\rho(x, t, y, s ; u) \sin \theta)(\sin \theta)^{2 \alpha-1} \mathrm{~d} \theta \Phi_{\kappa}(u) \mathrm{d} u .
\end{aligned}
$$

By (3.8), the coefficient of $r^{n}$ is the reproducing kernel $\mathrm{P}_{n}\left(\mathrm{w}_{\kappa} ; \cdot, \cdot\right)$, which gives the stated formula for $\alpha>0$. The proof for $\alpha=0$ follows the same procedure but using (2.4) instead. Finally, using $\rho(x, t, 0,0)=0$ and $j_{\alpha}(0)=1 /\left(2^{\alpha} \Gamma(\alpha+1)\right)$, (3.13) follows readily from (3.11) and (3.12). 


\section{Convolution structure on CONiC Surface With Laguerre Weight}

The closed form of the reproducing kernel suggests a pseudo convolution structure on the conic surface, which is bounded in the $L^{p}$ space defined on $\mathbb{V}_{0}^{d+1}$ as follows: $f \in L_{\mathrm{u}(\kappa)}^{p}\left(\mathbb{V}_{0}^{d+1}\right)$ if $\|f\|_{p, \kappa}$ is finite, where

$$
\|f\|_{p, \kappa}:=\left(\mathrm{b}_{\kappa} \int_{\mathbb{V}_{0}^{d+1}}\left|f(x, t) \mathrm{e}^{-t / 2}\right|^{p} \mathrm{u}_{\kappa}(x, t) \mathrm{d} \sigma(x, t)\right)^{\frac{1}{p}}, \quad \mathrm{u}_{k}(x, t)=t^{-1} h_{\kappa}^{2}(x),
$$

for $1 \leq p<\infty$, and

$$
\|f\|_{\infty, \kappa}:=\operatorname{ess} \sup \left\{|f(x, t)| \mathrm{e}^{-t / 2}:(x, t) \in \mathbb{V}_{0}^{d+1}\right\} .
$$

For $p=2$, the norm $\|\cdot\|_{2, \kappa}$ coincides with the usual norm of $L^{2}\left(\mathbb{V}_{0}^{d+1}, \mathrm{w}_{\kappa}\right)$.

The pseudo convolution is defined via a generalized translation operator, which is defined and shown to be bounded in the first subsection. The convolution structure is studied in the second subsection, which is used to analyze the Cesàro summability of the generalized Laguerre series on the conic surface in the third subsection.

4.1. Translation operator. For $d \geq 2, \kappa_{i} \geq 0$, let $\alpha_{\kappa}=|\kappa|+\frac{d-2}{2}$ as before. For $g: \mathbb{R}_{+} \mapsto \mathbb{R}$ we define a generalized translation operator $\mathrm{T}_{(x, t)}$.

Definition 4.1. Let $g \in L_{u\left(2 \alpha_{\kappa}\right)}^{1}\left(\mathbb{R}_{+}\right)$and $(x, y) \in \mathbb{V}_{0}^{d+1}$. If $\alpha_{k}>0$, define

$$
\begin{aligned}
\mathrm{T}_{(x, t)} g(y, s)= & C_{\kappa} \int_{[-1,1]^{d}} \int_{0}^{\pi} g(t+s+2 \rho(x, t, y, s ; u) \cos \theta) \\
& \times e^{-\rho(x, t, y, s ; u) \cos \theta} j_{\alpha_{\kappa}-1}(\rho(x, t, y, s ; u) \sin \theta)(\sin \theta)^{2 \alpha_{\kappa}-1} \mathrm{~d} \theta \Phi_{\kappa}(u) \mathrm{d} u,
\end{aligned}
$$

where $C_{\kappa}$ is given in (3.11); if $d=2$ and $\kappa=0$, define

$$
\begin{aligned}
\mathrm{T}_{(x, t)} g(y, s)= & \frac{1}{2}\left[\mathrm{e}^{-\rho(x, t, y, s)} g(t+s+2 \rho(x, t, y, s))+\mathrm{e}^{\rho(x, t, y, s)} g(t+s-2 \rho(x, t, y, s))\right] \\
& -\frac{1}{2} \rho(x, t, y, s)^{2} \int_{0}^{\pi} g(t+s+2 \rho(x, t, y, s) \cos \theta) \\
& \times e^{-\rho(x, t, y, s) \cos \theta} j_{1}(\rho(x, t, y, s) \sin \theta) \sin \theta \mathrm{d} \theta .
\end{aligned}
$$

The definition is motivated by the closed form formula of the reproducing kernel. Indeed, it follows readily that

$$
\mathrm{P}_{n}\left(\mathrm{w}_{\kappa} ;(x, t), \cdot\right)=\mathrm{T}_{(x, t)} L_{n}^{2|\kappa|+d-2}, \quad n=0,1,2, \ldots
$$

The generalized translation operator will be used to define a pseudo convolution structure on the conic surface in the next subsection. We first show that this operator is bounded. It turns out that we need to consider two separated cases.

Proposition 4.2. Let $d \geq 2$. Assume $\alpha_{k}=|\kappa|+\frac{d-2}{2} \geq \frac{1}{2}$. If $g \in L_{u\left(2 \alpha_{\kappa}\right)}^{p}\left(\mathbb{R}_{+}\right)$for $1 \leq p \leq \infty$, then

$$
\left\|\mathrm{T}_{(x, t)} g\right\|_{p, \kappa} \leq e^{t / 2}\|g\|_{p, u\left(2 \alpha_{\kappa}\right)}, \quad 1 \leq p \leq \infty .
$$

Proof. Setting $x=t \xi$ and $y=s \eta, \xi, \eta \in \mathbb{S}^{d-1}$, we can write

$$
\rho(x, t, y, s ; u)=\sqrt{t s} v(\xi, \eta ; u), \quad v(\xi, \eta ; u)=\sqrt{\frac{1}{2}\left(1+\xi_{1} \eta_{1} u_{1}+\cdots+\xi_{d} \eta_{d} u_{d}\right)} .
$$


To simplify the notation, we write $\alpha=\alpha_{\kappa}$ throughout the proof and define

$$
G(t, s ; z)=g(t+s+2 \sqrt{t s} z) \mathrm{e}^{-\sqrt{t s} z}
$$

The integral representation of the Bessel function [3, (10.9.4)] states that

$$
j_{\alpha}(z)=\frac{2^{-\alpha}}{\sqrt{\pi} \Gamma\left(\alpha+\frac{1}{2}\right)} \int_{-1}^{1} e^{\mathrm{i} z u}\left(1-u^{2}\right)^{\alpha-\frac{1}{2}} \mathrm{~d} u, \quad \alpha>-\frac{1}{2}
$$

and the formula holds under the limit when $\alpha \rightarrow-\frac{1}{2}+$, so that $j_{-\frac{1}{2}}(z)=\sqrt{\frac{2}{\pi}} \cos z$. It implies, in particular, that $\left|j_{\alpha-1}(z)\right| \leq 1 /\left(2^{\alpha-1} \Gamma(\alpha)\right)$ for all $z \in \mathbb{R}$ and $\alpha \geq \frac{1}{2}$. With the explicit formula of $C_{\kappa}$, it follows that $C_{\kappa}\left|j_{\alpha-1}(z)\right| \leq c_{\alpha-\frac{1}{2}} c_{\kappa-\frac{1}{2}}$. This last inequality leads to an upper bound of $\left|\mathrm{T}_{(x, t)} g\right|$,

$$
\begin{aligned}
\left|\mathrm{T}_{(x, t)} g(y, s)\right| & \leq c_{\alpha-\frac{1}{2}} c_{\kappa-\frac{1}{2}} \int_{[-1,1]^{d}} \int_{0}^{\pi}|G(t, s ; v(\xi, \eta ; u) \cos \theta)|(\sin \theta)^{2 \alpha-1} \mathrm{~d} \theta \Phi_{\kappa}(u) \mathrm{d} u \\
& =: F(t, \xi, s, \eta) .
\end{aligned}
$$

By the definition of $\|g\|_{\infty, \kappa}$, it follows readily that

$$
|G(t, s ; u)|=|g(t+s+2 \sqrt{t s} u)| \mathrm{e}^{-(t+s+2 \sqrt{t s} u) / 2} \mathrm{e}^{(t+s) / 2} \leq \mathrm{e}^{(t+s) / 2}\|g\|_{\infty, u\left(2 \alpha_{\kappa}\right)},
$$

which leads immediately to

$$
\left\|\mathrm{T}_{(x, t)} g\right\|_{\infty, \kappa}=\sup _{(y, s)}\left|\mathrm{T}_{(x, t)}(t, s, v(\xi, \eta))\right| \mathrm{e}^{-s / 2} \leq \mathrm{e}^{t / 2}\|g\|_{\infty, u\left(2 \alpha_{\kappa}\right)}
$$

This establishes the stated result for $p=\infty$.

Next we consider $p=1$. Using the intertwining operator $V_{\kappa}$, we can write

$$
F(t, \xi, s, \eta)=c_{\alpha-\frac{1}{2}} \int_{0}^{\pi} V_{\kappa}\left[\left|G\left(t, s ; \sqrt{\frac{1+\langle\xi, \cdot\rangle}{2}} \cos \theta\right)\right|\right](\eta)(\sin \theta)^{2 \alpha-1} \mathrm{~d} \theta .
$$

Hence, using the identity (2.16), we obtain that

$$
\begin{aligned}
I_{(x, t)}(s) & :=c_{\kappa}^{h} \int_{\mathbb{S}^{d-1}} F(t, \xi, s, \eta) h_{\kappa}^{2}(\eta) \mathrm{d} \sigma_{\mathbb{S}}(\eta) \\
& =c_{\alpha-\frac{1}{2}} \int_{0}^{\pi} c_{\alpha} \int_{-1}^{1}\left|G\left(t, s, \sqrt{\frac{1+u}{2}} \cos \theta\right)\right|\left(1-u^{2}\right)^{\alpha-\frac{1}{2}} \mathrm{~d} u(\sin \theta)^{2 \alpha-1} \mathrm{~d} \theta \\
& =2^{2 \alpha+1} c_{\alpha-\frac{1}{2}} c_{\alpha} \int_{0}^{\pi} \int_{0}^{1}|G(t, s, v \cos \theta)| v^{2 \alpha}\left(1-v^{2}\right)^{\alpha-\frac{1}{2}} \mathrm{~d} v(\sin \theta)^{2 \alpha-1} \mathrm{~d} \theta,
\end{aligned}
$$


where the second step follows from changing variable $v \rightarrow \sqrt{(1+u) / 2}$. Changing variable $\theta \mapsto u=v \cos \theta$ in the integral with respect to $\mathrm{d} \theta$, we further obtain that

$$
\begin{aligned}
I_{(x, t)}(s) & =2^{2 \alpha+1} c_{\alpha-\frac{1}{2}} c_{\alpha} \int_{0}^{1} \int_{-v}^{v}|G(t, s ; u)|\left(v^{2}-u^{2}\right)^{\alpha-1} \mathrm{~d} u v\left(1-v^{2}\right)^{\alpha-\frac{1}{2}} \mathrm{~d} v \\
& =2^{2 \alpha+1} c_{\alpha-\frac{1}{2}} c_{\alpha} \int_{-1}^{1}|G(t, s ; u)| \int_{|u|}^{1} v\left(v^{2}-u^{2}\right)^{\alpha-1}\left(1-v^{2}\right)^{\alpha-\frac{1}{2}} \mathrm{~d} v \mathrm{~d} u \\
& =2^{2 \alpha} c_{\alpha-\frac{1}{2}} c_{\alpha} \int_{-1}^{1}|G(t, s ; u)|\left(1-u^{2}\right)^{2 \alpha-\frac{1}{2}} \mathrm{~d} u \int_{0}^{1} z^{\alpha-1}(1-z)^{\alpha-\frac{1}{2}} \mathrm{~d} z \\
& =c_{2 \alpha} \int_{-1}^{1}|G(t, s ; u)|\left(1-u^{2}\right)^{2 \alpha-\frac{1}{2}} \mathrm{~d} u,
\end{aligned}
$$

where we have verified in the last step, using $\Gamma(2 a)=2^{2 a-1} \Gamma(a) \Gamma\left(a+\frac{1}{2}\right) / \sqrt{\pi}$, that

$$
2^{2 \alpha} c_{\alpha-\frac{1}{2}} c_{\alpha} \int_{0}^{1} z^{\alpha-1}(1-z)^{\alpha-\frac{1}{2}} \mathrm{~d} z=2^{2 \alpha} c_{\alpha-\frac{1}{2}} c_{\alpha} \frac{\Gamma(\alpha) \Gamma\left(\alpha+\frac{1}{2}\right)}{\Gamma\left(2 \alpha+\frac{1}{2}\right)}=c_{2 \alpha} .
$$

Changing one more variable $u \rightarrow z=t+s-2 \sqrt{t s} u$ in the last integral of (4.17), we further obtain

$$
\begin{aligned}
I_{(x, t)}(s) & =c_{2 \alpha} \int_{z_{-}}^{z_{+}}|g(z)| \mathrm{e}^{-\frac{z-t-s}{2}}\left(1-\frac{(z-t-s)^{2}}{4 t s}\right)^{2 \alpha-\frac{1}{2}} \frac{\mathrm{d} z}{2 \sqrt{t s}} \\
& =c_{2 \alpha} \int_{0}^{\infty}|g(z)| H_{\alpha}(t, s ; z) z^{2 \alpha} \mathrm{e}^{-z} \mathrm{~d} z,
\end{aligned}
$$

where $z_{ \pm}=t+s \pm 2 \sqrt{t s}=(\sqrt{t} \pm \sqrt{s})^{2}$ and

$$
H_{\alpha}(t, s ; z)=\frac{\left(t^{2}+s^{2}+z^{2}-2 t s-2 t z-2 s z\right)^{2 \alpha-\frac{1}{2}}}{(4 t s z)^{2 \alpha}} \mathrm{e}^{\frac{t+s+z}{2}},
$$

if $z \in\left[z_{-}, z_{+}\right]$and $H_{d}(t, s ; z)=0$ otherwise. In particular, it follows that $H_{\alpha}(t, s, z)=0$ if $2 t s+2 t z+2 s z \leq t^{2}+s^{2}+z^{2}$, so that $H_{\alpha}(t, s ; z)$ is symmetric in $t, s$ and $z$ and it is nonnegative. Moreover, choosing $g(z)=\mathrm{e}^{(-z+t+s) / 2}$ in the (4.18), it follows readily that

$$
c_{2 \alpha} \int_{0}^{\infty} H_{\alpha}(t, s ; z) z^{2 \alpha} \mathrm{e}^{-\frac{(z+t+s)}{2}} \mathrm{~d} z=c_{2 \alpha} \int_{-1}^{1}\left(1-u^{2}\right)^{2 \alpha-\frac{1}{2}} \mathrm{~d} u=1 .
$$

Consequently, exchanging the order of integrals and, by the symmetry of $H_{\alpha}(t, s ; z)$, integrating $H_{\alpha}(t, s ; z)$ with respect to $s$ first, we obtain by (4.18) and (4.19) that

$$
\begin{aligned}
\left\|\mathrm{T}_{(x, t)} g\right\|_{1, \kappa} & =\mathrm{b}_{\kappa} \int_{\mathbb{V}_{0}^{d+1}}\left|\mathrm{~T}_{(x, t)} g(y, s) \mathrm{e}^{-s / 2}\right| s^{-1} h_{\kappa}^{2}(y) \mathrm{d} \sigma(y, s) \\
& \leq b_{2 \alpha} \int_{0}^{\infty} I_{(x, t)}(s) s^{2 \alpha} \mathrm{e}^{-s / 2} \mathrm{~d} s \\
& =c_{2 \alpha} b_{2 \alpha} \int_{0}^{\infty}|g(z)| \int_{0}^{\infty} H_{\alpha}(t, s ; z) s^{2 \alpha} \mathrm{e}^{-(s+z) / 2} \mathrm{~d} s z^{2 \alpha} \mathrm{e}^{-z / 2} \mathrm{~d} z \\
& =\mathrm{e}^{t / 2} b_{2 \alpha} \int_{0}^{\infty}|g(z)| z^{2 \alpha} \mathrm{e}^{-z / 2} \mathrm{~d} z=\mathrm{e}^{t / 2}\|g\|_{1, u(2 \alpha)},
\end{aligned}
$$

which completes the proof for $p=1$. The case $1<p<\infty$ follows from the RieszThorin theorem by interpolating between the estimates for $p=1$ and $p=\infty$. This completes the proof. 
The operator $\mathrm{T}_{(x, t)}$ is not a positive operator because of the presence of the Bessel function, but its boundedness in the $L_{\mathrm{u}(\kappa)}^{p}$ norm is as strong as that of a positive operator in the sense that it is bounded with a constant 1 in the right-hand side of the inequality in the Proposition 4.2 .

The above proposition holds under the assumption that $\alpha_{\kappa} \geq \frac{1}{2}$, which comes from the upper bound of $j_{\alpha_{\kappa}-1}(t)$ deduced from (4.16). For $\alpha_{\kappa}<\frac{1}{2}$, we need to a different upper bound of $j_{\alpha_{\kappa}-1}$ given in the following lemma.

Lemma 4.3. For $\alpha>0$ and $t \in \in \mathbb{R}_{+}$,

$$
\left|j_{\alpha-\frac{1}{2}}(t)\right| \leq \frac{1}{2^{\alpha-1} \Gamma(\alpha)}+\frac{t^{-1}}{2^{\alpha} \sqrt{\pi} \Gamma\left(\alpha+\frac{3}{2}\right)} .
$$

Proof. We start from the three-term relation $J_{\alpha-1}(t)+J_{\alpha+1}(t)=2 \alpha t^{-1} J_{\alpha}(t)$ of the Bessel function [3, (10.6.1)], which gives immediately

$$
j_{\alpha-1}(t)=2 \alpha j_{\alpha}(t)-t^{2} j_{\alpha+1}(t), \quad \alpha>0 .
$$

We use (4.16) for $j_{\alpha+1}$ and integrate by parts once to obtain

$$
j_{\alpha+1}(t)=\frac{2^{-\alpha-1}}{\sqrt{\pi} \Gamma\left(\alpha+\frac{3}{2}\right)} \frac{2 \alpha+1}{i t} \int_{-1}^{1} \mathrm{e}^{\mathrm{i} t u} u\left(1-u^{2}\right)^{\alpha-\frac{1}{2}} \mathrm{~d} u
$$

which implies that $t\left|j_{\alpha+1}(t)\right| \leq \frac{2^{-\alpha}}{\sqrt{\pi} \Gamma\left(\alpha+\frac{3}{2}\right)}$. Hence, using $2 \alpha\left|j_{\alpha}(t)\right| \leq \frac{1}{2^{\alpha-1} \Gamma(\alpha)}$, the desired estimate follows from the three term relation.

Proposition 4.4. Let $d \geq 2$ and $\alpha_{\kappa}=|\kappa|+\frac{d-2}{2}<\frac{1}{2}$. For $g \in L_{u\left(2 \alpha_{\kappa}\right)}^{1}\left(\mathbb{R}_{+}\right) \cap$ $L_{u\left(2 \alpha_{\kappa}+\frac{1}{2}\right)}^{1}\left(\mathbb{R}_{+}\right)$,

$$
\left\|\mathrm{T}_{(x, t)} g\right\|_{1, \kappa} \leq e^{t / 2}\|g\|_{1, u\left(2 \alpha_{k}\right)}+\frac{1}{\left(2 \alpha_{\kappa}+1\right) \sqrt{\pi}} \sqrt{t} \mathrm{e}^{t / 2}\|g\|_{1, u\left(2 \alpha_{\kappa}+\frac{1}{2}\right)} .
$$

Proof. Let $\alpha=\alpha_{k}$. We need to consider two cases, $\alpha=0$ and $\alpha>0$. Assume first $\alpha>0$. We adopt the same notation as in the proof of the previous proposition for $\alpha \geq \frac{1}{2}$. Using Lemma 4.3. we obtain $C_{\kappa}\left|j_{\alpha-\frac{1}{2}}(z)\right| \leq c_{\alpha-\frac{1}{2}} c_{\kappa-\frac{1}{2}}+\frac{z}{(2 \alpha+1) \pi} c_{\kappa-\frac{1}{2}}$. Applying this inequality with $z=\rho(x, t, y, s ; u)=\sqrt{t s} v(\xi, \eta ; u)$, we obtain

$$
\left|\mathrm{\top}_{(x, t)} g(y, s)\right| \leq F(t, \xi, s, \eta)+F_{1}(t, \xi, s, \eta),
$$

where $F(t, \xi, s, \eta)$ is the same as before and $F_{1}(t, \xi, s, \eta)$ is defined by

$$
\begin{aligned}
F_{1}(t, \xi, s, \eta)=\frac{\sqrt{t s}}{(2 \alpha+1) \pi} \int_{0}^{\pi} V_{\kappa} & {\left[\sqrt{\frac{1+\langle\xi, \cdot\rangle}{2}}\left|G\left(t, s ; \sqrt{\frac{1+\langle\xi, \cdot\rangle}{2}} \cos \theta\right)\right|\right](\eta) } \\
& \times(\sin \theta)^{2 \alpha} \mathrm{d} \theta
\end{aligned}
$$

in terms of the intertwining operator $V_{\kappa}$. The estimate of $F(t, \xi, s, \eta)$ for $\alpha \geq \frac{1}{2}$ remains valid for $\alpha<\frac{1}{2}$, which gives the first term in the right-hand side of the desired estimate. The estimate of $F_{1}(t, \xi, s, \eta)$ can be carried out by the similar approach. In particular, following the proof of Proposition 4.2 and checking the constant carefully, we obtain

$$
\begin{aligned}
I_{(x, t)}(s) & :=c_{\kappa}^{h} \int_{\mathbb{S}^{d-1}} F_{1}(t, \xi, s, \eta) h_{\kappa}^{2}(\eta) d \sigma_{\mathbb{S}}(\eta) \\
& =\frac{\sqrt{t s}}{(2 \alpha+1) \pi} \int_{-1}^{1}|G(t, s ; u)|\left(1-u^{2}\right)^{2 \alpha} \mathrm{d} u .
\end{aligned}
$$


Changing variable $u \rightarrow z=t+s-2 \sqrt{t s} u$ as in the case of $\alpha_{\kappa} \geq \frac{1}{2}$, we further obtain

$$
I_{(x, t)}(s)=\frac{1}{(2 \alpha+1) \pi} \int_{0}^{\infty}|g(z)| H_{\alpha}^{(1)}(t, s ; z) z^{2 \alpha} \mathrm{e}^{-z} \mathrm{~d} z,
$$

where $H_{\alpha}^{(1)}$ is defined by

$$
H_{\alpha}^{(1)}(t, s ; z)=\frac{\left(t^{2}+s^{2}+z^{2}-2 t s-2 t z-2 s z\right)^{2 \alpha}}{2(4 t s z)^{2 \alpha}} \mathrm{e}^{\frac{t+s+z}{2}},
$$

if $z \in\left[z_{-}, z_{+}\right]$and $H_{d}(t, s ; z)=0$ otherwise. Again, $H_{\alpha}^{(1)}(t, s ; z)$ is symmetric in $t, s$ and $z$ and it is nonnegative. Furthermore, we also have

$$
\int_{0}^{\infty} H_{\alpha}(t, s ; z)^{(1)} z^{2 \alpha} \mathrm{e}^{-\frac{(z+t+s)}{2}} \mathrm{~d} z=\sqrt{t s} \int_{-1}^{1}\left(1-u^{2}\right)^{2 \alpha} \mathrm{d} u=\frac{\sqrt{t s}}{c_{2 \alpha+\frac{1}{2}}} .
$$

Consequently, continuing as in the proof of Proposition 4.2. we conclude that

$$
\begin{aligned}
\left\|F_{1}(t, \xi, \cdot)\right\|_{1, \kappa} & =b_{2 \alpha} \int_{0}^{\infty} I_{(x, t)}(s) s^{2 \alpha} \mathrm{e}^{-s / 2} \mathrm{~d} s \\
& =\frac{b_{2 \alpha}}{(2 \alpha+1) \pi} \int_{0}^{\infty}|g(z)| \int_{0}^{\infty} H_{\alpha}^{(1)}(t, s ; z) s^{2 \alpha} \mathrm{e}^{-(s+z) / 2} \mathrm{~d} s z^{2 \alpha} \mathrm{e}^{-z / 2} \mathrm{~d} z \\
& =\sqrt{t} \mathrm{e}^{t / 2} \frac{b_{2 \alpha}}{(2 \alpha+1) \pi c_{\alpha+\frac{1}{2}}} \int_{0}^{\infty}|g(z)| z^{2 \alpha+\frac{1}{2}} \mathrm{e}^{-z / 2} \mathrm{~d} z \\
& =\frac{1}{(2 \alpha+1) \sqrt{\pi}} \sqrt{t} \mathrm{e}^{t / 2}\|g\|_{1, u\left(2 \alpha+\frac{1}{2}\right)},
\end{aligned}
$$

where we have used $b_{2 \alpha}=1 / \Gamma(2 \alpha+1)$ and $1 / c_{2 \alpha+\frac{1}{2}}=b_{2 \alpha+\frac{1}{2}} \sqrt{\pi} / b_{2 \alpha}$ in the last step. This completes the proof for $\alpha>0$.

Next we consider $\alpha=0$, which is equivalent to $\kappa=0$ and $d=2$ since $|\kappa| \geq 0$ and $d \geq 2$. This corresponds to the Laguerre weight $\mathrm{w}_{0}(x, t)=t^{-1} \mathrm{e}^{-t}$ on the conic surface $\mathbb{V}_{0}^{3}$ of $\mathbb{R}^{3}$. In this case, we write the operator $T_{(x, t)}$ as a sum of two parts,

$$
\mathbf{T}_{(x, t)}=\mathbf{T}_{(x, t)}^{(1)}+\mathbf{T}_{(x, t)}^{(2)},
$$

where, using the notation $G(t, s ; u)$ and $v(\xi, \eta)=\sqrt{\frac{1}{2}(1+\langle\xi, \eta\rangle)}$, we have

$$
\begin{aligned}
& \mathbf{T}_{(x, t)}^{(1)} g(y, s)=\frac{1}{2}[G(t, s ; v(\xi, \eta))+G(t, s ;-v(\xi, \eta))] ; \\
& \mathbf{T}_{(x, t)}^{(2)} g(y, s)=-\frac{t s}{2}[v(\xi, \eta)]^{2} \int_{0}^{\pi} G(t, s ; v(\xi, \eta) \cos \theta) j_{1}(\sqrt{t s} v(\xi, \eta) \sin \theta) \sin \theta \mathrm{d} \theta .
\end{aligned}
$$

For $\mathrm{T}_{(x, t)}^{(1)} g$, we first integrate over $\mathbb{S}^{1}$, which is the unit circle and $\mathbf{b}_{2}=2 \pi$. Let $\langle\xi, \eta\rangle=\cos \phi$. Then $v(\xi, \eta)=\cos \frac{\phi}{2}$. Hence,

$$
\begin{aligned}
\frac{1}{2 \pi} \int_{\mathbb{S}^{1}}\left|\mathrm{~T}_{(x, t)}^{(1)} g(y, s)\right| d \sigma & =\frac{1}{4 \pi} \int_{0}^{2 \pi}\left|G\left(t, s ; \cos \frac{\phi}{2}\right)+G\left(t, s ;-\cos \frac{\phi}{2}\right)\right| \mathrm{d} \phi \\
& =\frac{1}{2 \pi} \int_{-1}^{1}|G(t, s ; u)+G(t, s ;-u)| \frac{\mathrm{d} u}{\sqrt{1-u^{2}}} \\
& \leq \frac{1}{\pi} \int_{-1}^{1}|G(t, s ; u)| \frac{\mathrm{d} u}{\sqrt{1-u^{2}}} .
\end{aligned}
$$


Sine $c_{0}=1 / \pi$, we see that the integral in the right-hand side is the integral in (4.17) when $\alpha_{k}=0$. The proof below (4.17) for $\alpha_{\kappa} \geq \frac{1}{2}$ remains valid for $\alpha_{\kappa}=0$, which proves the bound for $\left\|\mathbf{T}_{(x, t)}^{(1)} g\right\|_{1,0} \leq e^{t / 2}\|g\|_{1, u(0)}$. For $\mathbf{T}_{(x, t)}^{(2)}$, we use the bound $\left|z j_{1}(z)\right| \leq \frac{2}{\pi}$ in the proof of Lemma 4.3 to obtain

$$
\left|\mathrm{T}_{(x, t)}^{(2)} g(y, s)\right| \leq \frac{1}{\pi} \sqrt{t s}|v(\xi, \eta)| \int_{0}^{\pi}|G(t, s ; v(\xi, \eta) \cos \theta)| \mathrm{d} \theta .
$$

Since $V_{\kappa}$ becomes identity operator when $\kappa=0$, the right-hand side of the above expression coincides with $F_{1}(t, \xi, s, \eta)$ with $\alpha=0$. The evaluation of the norm of $F_{1}$ in the case of $\alpha>0$ remains valid for $\alpha=0$, so that the norm $\left\|\mathrm{T}_{(x, t)}^{(2)} g\right\|_{1,0} \leq \frac{1}{\sqrt{\pi}}\|g\|_{1, u\left(\frac{1}{2}\right)}$ follows right away. This completes the proof.

4.2. Pseudo convolution structure on the conic surface. We define a pseudo convolution structure on the conic surface.

Definition 4.5. Let $d \geq 2, \kappa_{i} \geq 0$ and $\alpha_{\kappa}=|\kappa|+\frac{d-2}{2}$. For $f \in L_{\mathrm{u}(\kappa)}^{1}\left(\mathbb{V}_{0}^{d+1}\right)$ and $g \in L_{u\left(2 \alpha_{\kappa}\right)}^{1}\left(\mathbb{R}_{+}\right)$, define

$$
f * g(x, t)=\mathrm{b}_{\kappa} \int_{\mathbb{V}_{0}^{d+1}} f(y, s) \boldsymbol{T}_{(x, t)} g(y, s) \mathrm{w}_{\kappa}(y, s) d \sigma(y, s), \quad(x, t) \in \mathbb{V}_{0}^{d+1} .
$$

From (4.15) it follows readily that, for $f \in L_{\mathbf{u}(\kappa)}^{2}\left(\mathbb{V}_{0}^{d+1}\right)$,

$$
\operatorname{proj}_{n}\left(\mathrm{w}_{\kappa} ; f\right)=f * L_{n}^{2|\kappa|+d-2}, \quad n=0,1,2, \ldots
$$

The following proposition justifies calling it a convolution.

Proposition 4.6. Let $d \geq 2$. For $f \in L^{2}\left(\mathbb{V}_{0}^{d+1}, \mathrm{w}_{\kappa}\right)$ and $g \in L^{2}\left(\mathbb{R}_{+}, w_{2 \alpha_{\kappa}}\right)$,

$$
\operatorname{proj}_{n}\left(\mathrm{w}_{\kappa} ; f * g\right)=\widehat{g}_{n}^{2 \alpha_{\kappa}} \operatorname{proj}_{n}\left(\mathrm{w}_{\kappa} ; f\right) \text {, }
$$

where $\widehat{g}_{n}^{2 \alpha_{\kappa}}$ is the Fourier-Laguerre coefficient as defined in (2.2). In particular,

$$
\|f * g\|_{2, k} \leq\|f\|_{2, \kappa}\|g\|_{2, u\left(2 \alpha_{\kappa}\right)} .
$$

Proof. Writing $g$ in terms of its Laguerre expansion in $L^{2}\left(\mathbb{R}_{+}, w_{2 \alpha_{\kappa}}\right)$ as in (2.2), it follows by (4.15) that

$$
\mathrm{T}_{(x, t)} g=\sum_{n=0}^{\infty} \widehat{g}_{n}^{2 \alpha_{\kappa}} \mathrm{P}_{n}((x, t), \cdot) \quad \text { and } \quad f * g=\sum_{n=0}^{\infty} \widehat{g}_{n}^{2 \alpha_{\kappa}} \operatorname{proj}_{n}\left(\mathrm{w}_{\kappa}\right),
$$

where the second identity follows from the first one by the definition of $f * g$. Since $\|\cdot\|_{2, \kappa}$ coincides with the norm of $L^{2}\left(\mathbb{V}_{0}^{d+1}, \mathrm{w}_{\kappa}\right)$ and $\|\cdot\|_{2, u(\alpha)}$ coincides with the norm of $L^{2}\left(\mathbb{R}_{+}, w_{\alpha}\right)$, we have the Parseval identities

$$
\|f * g\|_{2, k}^{2}=\sum_{n=0}^{\infty}\left\|\operatorname{proj}_{n}\left(\mathrm{w}_{\kappa} ; f * g\right)\right\|_{2, \kappa}^{2} \quad \text { and } \quad\|g\|_{2, u\left(\alpha_{\kappa}\right)}^{2}=\sum_{n=0}^{\infty}\left|\widehat{g}_{n}^{2 \alpha_{\kappa}}\right|^{2} .
$$

Hence, (4.23) follows from (4.22) and the Cauchy inequality.

We now show that the pseudo convolution operator is bounded in $L_{\mathrm{u}(\kappa)}^{p}\left(\mathbb{V}_{0}^{d+1}\right)$ for $p \neq 2$. We again need to consider two cases: $\alpha_{\kappa} \geq \frac{1}{2}$ and $\alpha_{k}<\frac{1}{2}$.

Theorem 4.7. Let $d \geq 2$. Assume $\alpha_{\kappa}=|\kappa|+\frac{d-2}{2} \geq \frac{1}{2}$. For $f \in L_{\mathrm{u}(\kappa)}^{p}\left(\mathbb{V}_{0}^{d+1}\right)$, $1 \leq p \leq \infty$, and $g \in L_{u\left(2 \alpha_{\kappa}\right)}^{1}\left(\mathbb{R}_{+}\right)$,

$$
\|f * g\|_{p, \kappa} \leq\|f\|_{p, \kappa}\|g\|_{1, u\left(2 \alpha_{\kappa}\right)}, \quad 1 \leq p \leq \infty .
$$


Proof. If $p=1$, then using the Proposition 4.2 and the obvious symmetry of $\mathrm{T}_{(x, t)}(y, s)$ in $(x, t)$ and $(y, s)$, we obtain

$$
\begin{aligned}
\|f * g\|_{1, \kappa} & =\mathrm{b}_{\kappa} \int_{\mathbb{V}_{0}^{d+1}}\left|\mathrm{~b}_{\kappa} \int_{\mathbb{V}_{0}^{d+1}} f(y, s) \mathbf{T}_{(x, t)} g(y, s) \mathbf{w}_{\kappa}(y, s) \mathrm{d} \sigma(y, s) \mathrm{e}^{-t / 2}\right| \mathbf{u}_{\kappa}(x, t) \mathrm{d} \sigma(x, t) \\
& \leq \mathbf{b}_{\kappa} \int_{\mathbb{V}_{0}^{d+1}}|f(y, s)| \cdot\left\|\mathbf{T}_{(y, s)} g\right\|_{\kappa, 1} \mathbf{w}_{\kappa}(y, s) \mathrm{d} \sigma(y, s) \\
& \leq\|g\|_{1, u\left(2 \alpha_{\kappa}\right)} \mathrm{b}_{\kappa} \int_{\mathbb{V}_{0}^{d+1}}|f(y, s)| e^{s / 2} \mathbf{w}_{\kappa}(y, s) \mathrm{d} \sigma(y, s) \\
& =\|f\|_{1, \kappa}\|g\|_{1, u\left(2 \alpha_{\kappa}\right)},
\end{aligned}
$$

which proves the stated inequality for $p=1$. Furthermore, for $p=\infty$,

$$
\begin{aligned}
\|f * g\|_{\infty, \kappa} & =\sup _{(x, t) \in \mathbb{V}_{0}^{d+1}}\left|f * g(x, t) \mathrm{e}^{-t / 2}\right| \\
& \leq\|f\|_{\infty, \kappa} \sup _{(x, t) \in \mathbb{V}_{0}^{d+1}} \mathrm{~b}_{\kappa} \int_{\mathbb{V}_{0}^{d+1}}\left|\mathrm{~T}_{(x, t)} g(y, s)\right| \mathrm{e}^{s / 2} \mathrm{w}_{\kappa}(y, s) \mathrm{d} \sigma(y, s) \mathrm{e}^{-t / 2} \\
& =\|f\|_{\infty, \kappa} \sup _{(x, t) \in \mathbb{V}_{0}^{d+1}}\left\|\mathrm{~T}_{(x, t)} g\right\|_{\kappa, 1} \mathrm{e}^{-t / 2} \leq\|f\|_{\infty, \kappa}\|g\|_{1, u\left(2 \alpha_{\kappa}\right)},
\end{aligned}
$$

which is the stated inequality for $p=\infty$. The case $1<p<\infty$ follows from the Riesz-Thorin interpolation theorem.

A straight forward generalization of the theorem is the Young's inequality.

Corollary 4.8. Let $d \geq 2$ and $\alpha_{\kappa} \geq \frac{1}{2}$. For $1 \leq p, q, r \leq \infty$ with $r^{-1}=p^{-1}+q^{-1}-1$, if $f \in L_{\mathrm{u}(\kappa)}^{p}\left(\mathbb{V}_{0}^{d+1}\right)$ and $g \in L_{u\left(2 \alpha_{\kappa}\right)}^{q}\left(\mathbb{R}_{+}\right)$, then $f * g \in L_{\mathrm{u}(\kappa)}^{r}\left(\mathbb{V}_{0}^{d+1}\right)$ and

$$
\|f * g\|_{r, \kappa} \leq\|f\|_{p, \kappa}\|g\|_{q, u\left(2 \alpha_{\kappa}\right)} .
$$

For the case $\alpha_{\kappa} \geq \frac{1}{2}$, the boundedness of $f * g$ is more restricted. We need to introduce another space $L_{\mathrm{u}(\kappa), *}^{p}\left(\mathbb{V}_{0}^{d+1}\right)$ that has the norm

$$
\|f\|_{p, \kappa}^{*}=\left(\mathrm{b}_{\kappa} \int_{\mathbb{V}_{0}^{d+1}}\left|f(x, t) e^{-t / 2}\right|^{p} \sqrt{t} \mathrm{u}_{\kappa}(x, t) \mathrm{d} \sigma(x, t)\right)^{\frac{1}{p}}, \quad 1 \leq p<\infty,
$$

which has an additional $\sqrt{t}$ in the integral in comparing with $\|f\|_{p, \kappa}$, and also

$$
\|f\|_{\infty, \kappa}^{*}=\operatorname{ess} \sup \left\{|f(x, t)| \sqrt{t} \mathrm{e}^{-t / 2}:(x, t) \in \mathbb{V}_{0}^{d+1}\right\} .
$$

Theorem 4.9. Let $d \geq 2$ and $\alpha_{\kappa}=|\kappa|+\frac{d-2}{2} \leq \frac{1}{2}$. For $f \in L_{\mathrm{u}(\kappa)}^{1}\left(\mathbb{V}_{0}^{d+1}\right) \cap L_{\mathrm{u}(\kappa), *}^{1}\left(\mathbb{V}_{0}^{d+1}\right)$ and $g \in L_{u\left(2 \alpha_{\kappa}\right)}^{1}\left(\mathbb{R}_{+}\right) \cap L_{u\left(2 \alpha_{\kappa}+\frac{1}{2}\right)}^{1}\left(\mathbb{R}_{+}\right)$,

$$
\|f * g\|_{1, \kappa} \leq\|f\|_{1, \kappa}\|g\|_{1, u\left(2 \alpha_{\kappa}\right)}+\|f\|_{1, \kappa}^{*}\|g\|_{1, u\left(2 \alpha_{\kappa}+\frac{1}{2}\right)} .
$$


Proof. The proof follows along the line of that Theorem 4.7 using Proposition 4.2 instead. It follows then that

$$
\begin{aligned}
\|f * g\|_{1, \kappa} \leq & \mathrm{b}_{\kappa} \int_{\mathbb{V}_{0}^{d+1}}|f(y, s)| \cdot\left\|\mathrm{T}_{(x, t)} g(y, s)\right\|_{1, \kappa} \mathrm{w}_{\kappa}(y, s) \mathrm{d} \sigma(y, s) \\
\leq & \|g\|_{1, u\left(2 \alpha_{\kappa}\right)} \mathrm{b}_{\kappa} \int_{\mathbb{V}_{0}^{d+1}}|f(y, s)| \mathrm{u}_{\kappa}(y, s) \mathrm{e}^{-s / 2} \mathrm{~d} \sigma(y, s) \\
& \quad+\frac{1}{\left(2 \alpha_{k}+1\right) \pi}\|g\|_{1, u\left(2 \alpha_{\kappa}+\frac{1}{2}\right)} \mathrm{b}_{\kappa} \int_{\mathbb{V}_{0}^{d+1}}|f(y, s)| \sqrt{s} \mathrm{u}_{\kappa}(y, s) \mathrm{e}^{-s / 2} \mathrm{~d} \sigma(y, s) \\
= & \|f\|_{1, \kappa}\|g\|_{1, u\left(2 \alpha_{k}\right)}+\frac{1}{\left(2 \alpha_{k}+1\right) \sqrt{\pi}}\|f\|_{1, \kappa}^{*}\|g\|_{1, u\left(2 \alpha_{k}+\frac{1}{2}\right)} .
\end{aligned}
$$

Since $\alpha_{\kappa} \geq 0$, we can replace the constant by 1 . This proves the stated inequality.

We restrict to the case $p=1$ in the above theorem. For $p=\infty$, we need to modify the the norm in the left-hand side. For example, the following inequality

$$
\left|f * g(x, t) \min \left\{1, t^{-\frac{1}{2}}\right\} \mathrm{e}^{-t / 2}\right| \leq\|f\|_{\infty, \kappa}\left(\|g\|_{\infty, u\left(2 \alpha_{k}\right)}+\|g\|_{\infty, u\left(2 \alpha_{k}+1\right)}\right) .
$$

follows readily. We omit the details.

4.3. Cesàro means on the conic surface. For $\delta>-1$, the Cesàro means of the Fourier orthogonal expansions with respect to $\mathrm{w}_{\kappa}$ is given by

$$
\begin{aligned}
\mathrm{S}_{n}^{\delta}\left(\mathrm{w}_{\kappa} ; f\right) & =\frac{1}{\left(\begin{array}{c}
n+\delta \\
n
\end{array}\right)} \sum_{k=0}^{n}\left(\begin{array}{c}
n-k+\delta \\
n-k
\end{array}\right) \operatorname{proj}_{k}\left(\mathrm{w}_{\kappa} ; f\right) \\
& =\mathrm{b}_{\kappa} \int_{\mathbb{V}_{0}^{d+1}} f(y, s) \mathrm{K}_{n}^{\delta}\left(\mathrm{w}_{\kappa} ;(x, t),(y, s)\right) \mathrm{w}_{\kappa}(y, s) \mathrm{d} \sigma(y, s),
\end{aligned}
$$

where $\mathrm{K}_{n}^{\delta}\left(\mathrm{w}_{\kappa}\right)$ denote the $(C, \delta)$ kernel

$$
\mathrm{K}_{n}^{\delta}\left(\mathrm{w}_{\kappa} ;(x, t),(y, s)\right)=\frac{1}{\left(\begin{array}{c}
n+\delta \\
n
\end{array}\right)} \sum_{k=0}^{n}\left(\begin{array}{c}
n-k+\delta \\
n-k
\end{array}\right) \mathrm{P}_{k}\left(\mathrm{w}_{\kappa} ;(x, t),(y, s)\right) .
$$

By the closed formula of the projection operator in (4.21), we can express the means in terms of the puedo convolution.

Proposition 4.10. Let $d \geq 2$ and $\delta>-1$. Then

$$
\mathrm{K}_{n}^{\delta}\left(\mathrm{w}_{\kappa} ;(x, t),(y, s)\right)=\frac{1}{\left(\begin{array}{c}
n+\delta \\
n
\end{array}\right)} \mathbf{T}_{(x, t)}\left(L_{n}^{\delta+2|\kappa|+d-1}\right)(y, s),
$$

and

$$
\mathrm{S}_{n}^{\delta}\left(\mathrm{w}_{\kappa} ; f\right)=\frac{1}{\left(\begin{array}{c}
n+\delta \\
n
\end{array}\right)} f * L_{n}^{\delta+2|\kappa|+d-1} .
$$

Proof. By (4.15), we see that

$$
\mathrm{K}_{n}\left(\mathrm{w}_{\kappa} ;(x, t), \cdot\right)=\mathrm{T}_{(x, t)} \tau_{n}^{\delta} \quad \text { with } \quad \tau_{n}^{\delta}=\frac{1}{\left(\begin{array}{c}
n+\delta \\
n
\end{array}\right)} \sum_{k=0}^{n}\left(\begin{array}{c}
n-k+\delta \\
n-k
\end{array}\right) L_{k}^{2|\kappa|+d-2} .
$$


From the identity (2.5) and the generating function (2.1), it follows that

$$
\begin{aligned}
\sum_{n=0}^{\infty}\left(\begin{array}{c}
n+\delta \\
n
\end{array}\right) \tau_{n}^{\delta}(z) & =\frac{1}{(1-r)^{\delta+1}} \sum_{n=0}^{\infty} L_{n}^{2|\kappa|+d-2}(z) r^{n} \\
& =\frac{1}{(1-r)^{\delta+2|\kappa|+d}} \mathrm{e}^{-\frac{z r}{1-r}}=\sum_{n=0}^{\infty} L_{n}^{\delta+2|\kappa|+d-1}(z) r^{n}
\end{aligned}
$$

Hence, comparing the coefficients of $r^{n}$, we obtain

$$
\tau_{n}^{\delta}=\frac{1}{\left(\begin{array}{c}
n+\delta \\
n
\end{array}\right)} L_{n}^{\delta+2|\kappa|+d-1}
$$

which proves (4.24) and, consequently, (4.25).

For $1 \leq p \leq \infty$, let $\left\|\mathrm{S}_{n}^{\delta}\left(\mathrm{w}_{\kappa}\right)\right\|_{p, \kappa}$ denote the operator norm of $\mathrm{S}_{n}^{\delta}$ defined by

$$
\left\|\mathrm{S}_{n}^{\delta}\left(\mathrm{w}_{\kappa}\right)\right\|_{p, \kappa}=\sup \left\{\left\|\mathrm{S}_{n}^{\delta}\left(\mathrm{w}_{\kappa} ; f\right)\right\|_{p, \kappa}: f \in L_{\mathrm{u}(\kappa)}^{p}\left(\mathbb{V}_{0}^{d+1}\right)\right\} .
$$

Theorem 4.11. Let $d \geq 2$ and $\delta>-1$. If $\alpha_{\kappa}=|\kappa|+\frac{d-2}{2} \geq \frac{1}{2}$, then for $p=1$ and $p=\infty$,

$$
\left\|S_{n}^{\delta}\left(\mathrm{w}_{\kappa}\right)\right\|_{p, \kappa}=\frac{1}{\left(\begin{array}{c}
n+\delta \\
n
\end{array}\right)}\left\|L_{n}^{\delta+2 \alpha_{k}+1}\right\|_{1, u\left(2 \alpha_{\kappa}\right)} \sim \begin{cases}n^{2 \alpha_{\kappa}+\frac{1}{2}-\delta} & -1<\delta<2 \alpha_{\kappa}+\frac{1}{2} \\
\log n & \delta=2 \alpha_{\kappa}+\frac{1}{2} \\
1 & \delta>2 \alpha_{\kappa}+\frac{1}{2} .\end{cases}
$$

Moreover, $\mathrm{S}_{n}^{\delta} f$ converse to $f$ in $L_{\mathrm{u}(\kappa)}^{p}\left(\mathbb{V}_{0}^{d+1}\right), 1 \leq p \leq \infty$, if $\delta>2 \alpha_{\kappa}+\frac{1}{2}=2|\kappa|+d-\frac{3}{2}$ and the condition is sharp if $p=1$ and $p=\infty$.

Proof. Let $\alpha_{\kappa}=|\kappa|+\frac{d-2}{2}$. We need to consider two cases $\alpha_{\kappa}>0$ and $\alpha_{\kappa}=0$ separately. Since $\mathrm{S}_{n}^{\delta}\left(\mathrm{w}_{\kappa} ; f\right)$ is an integral operator, a standard argument shows that,

$$
\left\|\mathrm{S}_{n}^{\delta}\left(\mathrm{w}_{\kappa}\right)\right\|_{p, \kappa}=\sup _{(x, t) \in \mathbb{V}_{0}^{d+1}} \int_{\mathbb{V}_{0}^{d+1}}\left|\mathrm{~K}_{n}^{\delta}\left(\mathrm{w}_{\kappa} ;(x, t),(y, s)\right)\right| \mathrm{e}^{s / 2} \mathbf{W}_{\kappa}(y, s) \mathrm{d} \sigma(y, s)
$$

for $p=1$ and $p=\infty$. By the estimate in Proposition 4.2 or Theorem 4.7, we have

$$
\left\|\mathrm{S}_{n}^{\delta}\left(\mathrm{w}_{\kappa}\right)\right\|_{p, \kappa} \leq \frac{1}{\left(\begin{array}{c}
n+\delta \\
n
\end{array}\right)}\left\|L_{n}^{\delta+2 \alpha_{k}+1}\right\|_{1, u\left(2 \alpha_{\kappa}\right)} .
$$

Furthermore, by (3.13), we see that $\mathrm{K}_{n}^{\delta}\left(\mathrm{w}_{\kappa} ;(0,0),(y, s)\right)=\frac{1}{\left(\begin{array}{c}n+o \\ n\end{array}\right)} L_{n}^{\delta+2 \alpha_{k}+1}(t)$, so that

$$
\begin{aligned}
\left\|\mathrm{S}_{n}^{\delta}\left(\mathrm{w}_{\kappa}\right)\right\|_{p, \kappa} & \geq \mathrm{b}_{\kappa} \int_{\mathbb{V}_{0}^{d+1}}\left|\mathrm{~K}_{n}^{\delta}\left(\mathrm{w}_{\kappa} ;(0,0),(y, s)\right)\right| \mathrm{w}_{\kappa}(y, s) \mathrm{e}^{s / 2} \mathrm{~d} \sigma(y, s) \\
& =\frac{1}{\left(\begin{array}{c}
n+\delta \\
n
\end{array}\right)} \int_{0}^{\infty}\left|L_{n}^{\delta+2 \alpha_{\kappa}+1}(t)\right| t^{2 \alpha_{\kappa}} \mathrm{e}^{-t / 2} \mathrm{~d} t .
\end{aligned}
$$

Together, we have proved the equality in (4.26), whereas the asymptotic in (4.26) follows form Lemma 2.1 with $\alpha=4 \alpha_{k}$ and $\beta=\delta-2 \alpha_{\kappa}+1$ and $\left(\begin{array}{c}n+\delta \\ n\end{array}\right) \sim n^{\delta}$. Consequently, it follows that $\left\|\mathrm{S}_{n}^{\delta} f\left(\mathrm{w}_{\kappa}\right)\right\|_{p, \kappa}$ is bounded if $\delta>2 \alpha_{\kappa}+\frac{1}{2}$ and it is unbounded if $\delta \leq 2 \alpha_{\kappa}+\frac{1}{2}$. This completes the proof.

It is evident that the lower bound of $\left\|\mathrm{S}_{n}^{\delta}\left(\mathrm{w}_{\kappa}\right)\right\|_{p, \mathrm{w}_{\kappa}}$ in (4.27) remains valid when $\alpha_{\kappa}<\frac{1}{2}$. However, the bound in Propostion 4.4 or Theorem 4.9 does not lead to a matching upper bound. 
Theorem 4.12. Let $d \geq 2$ and $\alpha_{\kappa} \leq \frac{1}{2}$. Then $\mathrm{S}_{n}^{\delta}\left(\mathrm{w}_{0} ; f\right)$ converse to $f$ in $L_{\mathrm{u}(\kappa)}^{1}\left(\mathbb{V}_{0}^{d+1}\right) \cap$ $L_{\mathrm{u}(\kappa), *}^{1}\left(\mathbb{V}_{0}^{d+1}\right)$ if $\delta>2 \alpha_{\kappa}+1$. Moreover, $\mathrm{S}_{n}^{\delta}\left(\mathrm{w}_{0} ; f\right)$ does not converge for all $f \in$ $L_{\mathrm{u}(\kappa)}^{1}\left(\mathbb{V}_{0}^{d+1}\right)$ if $\delta \leq 2 \alpha_{\kappa}+\frac{1}{2}$.

Proof. Using Theorem 4.9, we obtain that

$$
\left\|\mathrm{S}_{n}^{\delta}\left(\mathrm{w}_{0} ; f\right)\right\|_{1, \kappa} \leq \frac{1}{\left(\begin{array}{c}
n+\delta \\
n
\end{array}\right)}\left(\left\|L_{n}^{\delta+2 \alpha_{\kappa}+1}\right\|_{1, u\left(2 \alpha_{\kappa}\right)}\|f\|_{1, \kappa}+\left\|L_{n}^{\delta+2 \alpha_{\kappa}+1}\right\|_{1, u\left(2 \alpha_{\kappa}+\frac{1}{2}\right)}\|f\|_{1, \kappa}^{*}\right) .
$$

The term $\frac{1}{\left(\begin{array}{c}n+\delta \\ n\end{array}\right)}\left\|L_{n}^{\delta+2 \alpha_{\kappa}+1}\right\|_{1, u\left(2 \alpha_{\kappa}\right)}$ is bounded as shown in the case of $\alpha_{\kappa} \geq \frac{1}{2}$. By Lemma 2.1 with $\alpha=4 \alpha_{k}+1$ and $\beta=\delta-2 \alpha_{k} 1$, we obtain

$$
\frac{1}{\left(\begin{array}{c}
n+\delta \\
n
\end{array}\right)}\left\|L_{n}^{\delta+1}\right\|_{1, u\left(2 \alpha_{k}+\frac{1}{2}\right)} \sim \begin{cases}n^{2 \alpha_{\kappa}+1-\delta} & \delta<2 \alpha_{k}+\frac{3}{2} \\
n^{2 \alpha_{\kappa}+1-\delta} \log n & \delta=2 \alpha_{k}+\frac{3}{2} \\
n^{-\frac{1}{2}} & \delta>2 \alpha_{k}+\frac{3}{2},\end{cases}
$$

which is bounded if $\delta>2 \alpha_{k}+1$. This proves the convergence part.

If $\kappa=0$, then our result holds for $\mathrm{w}_{0}(x, t)=t^{-1} \mathrm{e}^{-t}$. In this case, our result shows that the $(C, \delta)$ means converge in $L_{\mathrm{u}(\kappa)}^{p}\left(\mathbb{V}_{0}^{d+1}\right), p=1$ and $\infty$ if and only if $\delta>d-\frac{3}{2}$ when $d \geq 3$. For $d=2$, the condition $\delta>\frac{1}{2}$ remains necessary but our sufficient condition $\delta>1$ is weaker. We believe that the condition $\delta>\frac{1}{2}$ should be sufficient as well, for which one may need to find a way to estimate the Cesàro kernel without using the closed form formula.

More generally, we believe that Theorem 4.11 should hold for all $\alpha_{\kappa} \geq 0$, which means that $\delta>2 \alpha_{\kappa}+\frac{1}{2}$ is necessary and sufficient. This is non-trivial even for the classical Laguerre expansions in $\mathbb{R}_{+}$, where the convergence of the $(C, \delta)$ means for $w_{\alpha}$ is determined via the convolution structure when $\alpha \geq 0$, but requires delicate hard estimate when $\alpha<0$. The same obstacle appears in the Laguerre expansions in $\mathbb{R}_{+}^{d}$ with the weight $w_{\alpha_{1}}\left(x_{1}\right) \cdots w_{\alpha_{d}}\left(x_{d}\right)$, for which the convergence of the $(C, \delta)$ means is determined under the restriction $\alpha_{i} \geq 0$ [18, 21] and, as far as we are aware, the case of negative $\alpha_{i}$ remains open.

The product Laguerre expansions of $\mathbb{R}_{+}^{2}$ is particularly pertinent to our $w_{0}$ weight when $d=2$. Indeed, as we shall see in the next section, the orthogonal expansion for $\mathrm{w}_{0}$ and $d=2$ is closely related to the orthogonal expansion on the solid cone $\mathbb{V}^{2}=\left\{(x, t) \in \mathbb{R}^{2}:|x| \leq t\right\}$ with respect to the weight function $\left(t^{2}-x^{2}\right)^{-\frac{1}{2}}$, and the latter is equivalent to the product Laguerre expansions with respect to the weight function $w_{-\frac{1}{2}}\left(x_{1}\right) w_{-\frac{1}{2}}\left(x_{2}\right)$ on $\mathbb{R}_{+}^{2}$; see Remark [5.1.

\section{LAGUERRE EXPANSIONS ON THE SOLID CONE}

In this section we consider the Laguerre expansion on the solid cone

$$
\mathbb{V}^{d+1}=\left\{(x, t) \in \mathbb{R}^{d+1}:\|x\| \leq t, x \in \mathbb{R}^{d}, t \in \mathbb{R}_{+}\right\}, \quad d \geq 1
$$

with respect to the weight function $W_{\kappa, \mu}$ defined by

$$
W_{\kappa, \mu}(x, t)=h_{\kappa}^{2}(x)\left(t^{2}-\|x\|^{2}\right)^{\mu-\frac{1}{2}} \mathrm{e}^{-t}, \quad \kappa_{i} \geq 0, \quad \mu>-\frac{1}{2} .
$$


5.1. Orthogonal polynomials. We define the inner product on $L^{2}\left(\mathbb{V}^{d+1}, W_{\kappa, \mu}\right)$ with respect to $W_{\kappa, \mu}$ by

$$
\langle f, g\rangle_{\kappa, \mu}=\mathbf{b}_{k, \mu} \int_{\mathbb{V}^{d+1}} f(x, t) g(x, t) W_{\kappa, \mu}(x, t) \mathrm{d} x \mathrm{~d} t,
$$

where $\mathbf{b}_{\kappa, \mu}=b_{2|\kappa|+2 \mu+d-1} b_{\kappa, \mu}^{\mathbb{B}}$ with $b_{\mu}^{\mathbb{B}}$ being the normalization constant of the weight $h_{\kappa}^{2}(x)\left(1-\|x\|^{2}\right)^{\mu-\frac{1}{2}}$ on $\mathbb{B}^{d}$. Let $\mathcal{V}_{n}\left(\mathbb{V}^{d+1}, W_{\kappa, \mu}\right)$ denote the space of orthogonal polynomials of degree $n$ with respect to this inner product. Then

$$
\operatorname{dim} \mathcal{V}_{n}\left(\mathbb{V}^{d+1}, W_{\mu}\right)=\left(\begin{array}{c}
n+d \\
n
\end{array}\right) .
$$

An orthogonal basis for this space can be given in terms of orthogonal polynomials on the unit ball and the Laguerre polynomials.

Proposition 5.1. Let $\left\{P_{\mathbf{k}}^{m}\left(\varpi_{\kappa, \mu}\right):|\mathbf{k}|=n\right\}$ be an orthonormal basis of $\mathcal{V}_{n}\left(\mathbb{B}^{d}, \varpi_{\kappa, \mu}\right)$. Define

$$
\mathbf{L}_{m, \mathbf{k}}^{n}(x, t)=L_{n-m}^{2 m+2|\kappa|+2 \mu+d-1}(t) t^{m} P_{\mathbf{k}}^{m}\left(\varpi_{\kappa, \mu} ; \frac{x}{t}\right), \quad|\mathbf{k}|=m, \quad 0 \leq m \leq n .
$$

Then $\left\{\mathbf{L}_{m, \mathbf{k}}^{n}:|\mathbf{k}|=m, \quad 0 \leq m \leq n\right\}$ is an orthogonal basis of $\mathcal{V}_{n}\left(\mathbb{V}^{d+1}, W_{\kappa, \mu}\right)$. Moreover, the norm square of $L_{m, \mathbf{k}}^{n}$ is given by

$$
\mathbf{h}_{m, n}^{\mathbf{L}}:=\left\langle\mathbf{L}_{m, \mathbf{k}}^{n}, \mathbf{L}_{m, \mathbf{k}}^{n}\right\rangle_{\kappa, \mu}=\frac{(2|\kappa|+2 \mu+d)_{n+m}}{(n-m) !} .
$$

The orthogonality is stated in 22, Proposition 3.3] and the norm can be verified directly as in the proof of Proposition 5.1. When $\kappa=0$, the polynomials $\mathbf{L}_{m, \ell}^{n}$ are called the Laguerre polynomials on the cone.

Remark 5.1. When $d=1$, the cone $\mathbb{V}_{0}^{2}$ is the wedge domain bounded by the two lines $x= \pm t$ in $\mathbb{R}^{2}$ and the weight function is $W_{\kappa, \mu}(x, t)=|x|^{2 \kappa}\left(t^{2}-x^{2}\right)^{\mu-\frac{1}{2}} \mathrm{e}^{-t}$, where we have written $\kappa=\kappa_{1} \geq 0$. If we rotate the domain by $90^{\circ}$ by setting $x_{1}=\frac{t+x}{2}$ and $x_{2}=\frac{t-x}{2}$, then the wedge domain becomes $\mathbb{R}_{+}^{2}$ and the weight function $W_{\kappa, \mu}$ becomes

$$
U_{\kappa, \mu}\left(x_{1}, x_{2}\right)=\left|x_{1}-x_{2}\right|^{2 \kappa}\left|x_{1} x_{2}\right|^{\mu-\frac{1}{2}} \mathrm{e}^{-x_{1}-x_{2}}, \quad\left(x_{1}, x_{2}\right) \in \mathbb{R}_{+}^{2} .
$$

In particular, for $\kappa=0, U_{\kappa, \mu}\left(x_{1}, x_{2}\right)=w_{\mu-\frac{1}{2}}\left(x_{1}\right) w_{\mu-\frac{1}{2}}\left(x_{2}\right)$ is the product Laguerre weight.

For $f \in L^{2}\left(\mathbb{V}^{d+1}, W_{\kappa, \mu}\right)$, the Fourier-Laguerre expansion on the cone is defined by

$$
f=\sum_{n=0}^{\infty} \sum_{m=0}^{n} \sum_{|\mathbf{k}|=m} \widehat{f}_{m, \mathbf{k}}^{n} \mathbf{L}_{m, \mathbf{k}}^{n} \quad \text { with } \quad \widehat{f}_{m, \mathbf{k}}^{n}=\frac{\left\langle f, \mathbf{L}_{m, \mathbf{k}}^{n}\right\rangle_{\mu}}{\mathbf{h}_{m, n}^{\mathbf{L}}}
$$

For $\kappa=0$, this orthogonal expansion can be used to derive an explicit solution for the non-homogeneous wave equation; see [13. The projection operator $\operatorname{proj}_{n}$ : $L^{2}\left(\mathbb{V}^{d+1} ; W_{\kappa, \mu}\right) \mapsto \mathcal{V}_{n}^{d}\left(\mathbb{V}^{d+1}, W_{\kappa, \mu}\right)$ and the $n$-th partial sum operator $\mathbf{S}_{n} f$ of this expansion are defined by

$$
\operatorname{proj}_{n}\left(W_{\kappa, \mu} ; f\right)=\sum_{m=0}^{n} \sum_{|\mathbf{k}|=m} \widehat{f}_{m, \mathbf{k}}^{n} \mathbf{L}_{m, \mathbf{k}}^{n} \quad \text { and } \quad \mathbf{S}_{n} f\left(W_{\kappa, \mu} ; f\right)=\sum_{k=0}^{n} \operatorname{proj}_{k}\left(W_{\kappa, \mu} ; f\right) .
$$

If $f(x, t)$ depends only on $t$, then $\widehat{f}_{m, \mathbf{k}}^{n}=0$ for all $m>0$, and the series is again reduced to the classical Fourier-Laguerre series. 
Proposition 5.2. Let $d \geq 1, \kappa \geq 0$ and $\mu \geq-\frac{1}{2}$. Define $\alpha=\mu+|\kappa|+\frac{d-1}{2}$. If $f(x, t)=f_{0}(t)$, where $f_{0} \in L^{2}\left(\mathbb{R}_{+} ; w_{2 \alpha}\right)$. Then the Fourier-Laguerre series of $f$ on the cone is equal to the Fourier-Laguree series of $f_{0}$ in $L^{2}\left(\mathbb{R}_{+} ; w_{2 \alpha}\right)$. In particular,

$$
\mathbf{S}_{n}\left(W_{\kappa, \mu} ; f,(x, t)\right)=s_{n}\left(w_{2 \alpha} ; f_{0}, t\right), \quad n=0,1,2, \ldots
$$

As in the case of conic surface, the polynomials $\mathbf{L}_{m, \mathbf{k}}^{n}$ can also be deduced from taking the limit of the Jacobi type orthogonal polynomials defined in [22] on the solid cone. A more direct way, however, is to relate them to the orthogonal polynomials on the conic surface that we have already encountered. The relation is modeled after, in fact uses, the relation between orthogonal polynomials on the unit ball and those on the unit sphere.

For $(x, t) \in \mathbb{V}^{d+1}$, we introduce the notation $X=\left(x, \sqrt{t^{2}-\|x\|^{2}}\right)$, so that $\|X\|=t$ and $(X, t) \in \mathbb{V}_{0}^{d+2}$. Similarly, for $(y, s) \in \mathbb{V}^{d+1}$, we let $Y=\left(y, \sqrt{t^{2}-\|y\|^{2}}\right) \in \mathbb{V}_{0}^{d+2}$. Define $\boldsymbol{\kappa}=(\kappa, \mu) \in \mathbb{R}^{d+2}$. Then, for $x=t x^{\prime}, x^{\prime} \in \mathbb{B}^{d}$, we can write

$$
W_{\kappa, \mu}(x, t) \mathrm{d} x \mathrm{~d} t=h_{\kappa}^{2}(X) t^{-1} e^{-t} \frac{\mathrm{d} x^{\prime} \mathrm{d} t}{\sqrt{1-\left\|x^{\prime}\right\|^{2}}}=\mathbf{w}_{\mathbf{k}}(X) \mathrm{d} \sigma(X, t),
$$

where $\mathrm{d} \sigma=\mathrm{d} \sigma_{\mathbb{V}_{0}^{d+2}}$ is the Lebesgue measure on the surface $\mathbb{V}_{0}^{d+2}$ in the right-hand side. Let

$$
\mathbb{V}_{0,+}^{d+2}=\left\{\left(x, x_{d+1}, t\right) \in \mathbb{V}_{0}^{d+2}: x_{d+1} \geq 0\right\}
$$

which is half of the conic surface $\mathbb{V}_{0}^{d+2}$. Likewise, we define $\mathbb{V}_{0,-}^{d+2}$ as the other half with $x_{d+1}<0$. Let $X^{-}=\left(x,-\sqrt{t^{2}-\|x\|^{2}}\right)$. Then $(x, t) \mapsto(X, t)$ maps $\mathbb{V}^{d+1}$ onto $\mathbb{V}_{0,+}^{d+2}$ and $(x, t) \mapsto\left(X^{-}, t\right)$ maps $\mathbb{V}^{d+1}$ onto $\mathbb{V}_{0,-}^{d+2}$. By symmetry, it follows that

$$
\int_{\mathbb{V}_{0}^{d+2}} f(y, s) \mathrm{w}_{\kappa}(y, s) \mathrm{d} \sigma(y, s)=\int_{\mathbb{V}^{d+1}} \frac{1}{2}\left[f(X, t)+f\left(X^{-}, t\right)\right] W_{\kappa, \mu}(X, t) \mathrm{d} x \mathrm{~d} t .
$$

As a consequence of this relation, we obtain a relation between orthogonal polynomials on the cone and the conic surface. To emphasis the dependence on the weight function, we denote the orthogonal polynomial given in (3.2) by $\mathbf{L}_{m, \mathbf{k}}^{n}\left(W_{\kappa, \mu}\right)$. Define

$$
Y_{m, \mathbf{k}}^{n, 1}(X, t)=\mathbf{L}_{m, \mathbf{k}}^{n}\left(W_{\kappa, \mu} ;(x, t)\right), \quad Y_{m, \mathbf{k}}^{n, 2}(X, t)=X_{d+1} \mathbf{L}_{m, \mathbf{k}}^{n-1}\left(W_{\kappa, \mu+1} ;(x, t)\right)
$$

for $X=\left(x, X_{d+1}\right) \in \mathbb{R}^{d+1}$ and $(X, t) \in \mathbb{V}_{0}^{d+2}$.

Proposition 5.3. Let $\boldsymbol{\kappa}=(\kappa, \mu)$ and $X=\left(x, X_{d+1}\right), X_{d+1}=\sqrt{t^{2}-\|x\|^{2}}$. Under the mapping $\mathbb{V}^{d+1} \mapsto \mathbb{V}_{0,+}^{d+2}:(x, t) \mapsto(X, t)$,

$$
\mathcal{V}_{n}\left(\mathbb{V}_{0}^{d+2}, \mathrm{w}_{\kappa}\right)=\mathcal{V}_{n}\left(\mathbb{V}_{0}^{d+1}, W_{\kappa, \mu}\right) \bigoplus X_{d+1} \mathcal{V}_{n}\left(\mathbb{V}_{0}^{d+1}, W_{\kappa, \mu+1}\right)
$$

More precisely, an orthogonal basis of $\mathcal{V}_{n}\left(\mathbb{V}_{0}^{d+2}, \mathrm{w}_{\kappa}\right)$ is given by

$$
\left\{Y_{\mathbf{k}, m}^{n, 1}:|\mathbf{k}|=m, 0 \leq m \leq n\right\} \cup\left\{Y_{\mathbf{k}, m}^{n, 2}:|\mathbf{k}|=m, 0 \leq m \leq n-1\right\} .
$$

Proof. Since $P_{\mathbf{k}}^{m}$ is even when $m$ is even and odd when $m$ is odd, it follows that $t^{m} P_{\mathbf{k}}^{m}\left(\frac{x}{t}\right)$ is a homogeneous polynomial of degree $m$ in the variables $(x, t)$ and, in particular, a polynomials of degree $m$ in the variables $(X, t)$. Hence, it follows that $Y_{\mathbf{k}, m}^{n, 1}$ is a polynomial of degree $n$ in $(X, t)$ variables, even with respect to $X_{d+1}$ variable. Moreover, the same argument shows that $Y_{\mathbf{k}, m}^{n, 2}$ is a polynomial of degree $n$ in $(X, t)$ variables, odd in $X_{d+1}$ variable. By (5.4), it follows that $Y_{\mathbf{k}, m}^{n, 1}$ and $Y_{\mathbf{k}, m}^{n, 2}$ 
are orthogonal with respect to $\langle\cdot, \cdot\rangle_{\kappa}$ on $\mathbb{V}_{0}^{d+2}$ by parity and, moreover, by (5.3) and $W_{\kappa, \mu+1}(x, t) \mathrm{d} x \mathrm{~d} t=X_{d+1}^{2} \mathrm{w}_{\mathbf{k}}(X) \mathrm{d} \sigma(X, t)$ followed by (5.3),

$$
\begin{aligned}
\left\langle Y_{\mathbf{k}, m}^{n, 2}, Y_{\mathbf{k}^{\prime}, m^{\prime}}^{n^{\prime}, 2}\right\rangle_{\kappa} & =\left\langle\mathbf{L}_{m, \mathbf{k}}^{n}\left(W_{\kappa, \mu}\right), \mathbf{L}_{m^{\prime}, \mathbf{k}^{\prime}}^{n^{\prime}}\left(W_{\kappa, \mu}\right)\right\rangle_{\kappa, \mu} \\
\left\langle Y_{\mathbf{k}, m}^{n, 1}, Y_{\mathbf{k}^{\prime}, m^{\prime}}^{n^{\prime}, 1}\right\rangle_{\boldsymbol{\kappa}} & =\left\langle\mathbf{L}_{m, \mathbf{k}}^{n-1}\left(W_{\kappa, \mu+1}\right), \mathbf{L}_{m^{\prime}, \mathbf{k}^{\prime}}^{n^{\prime}-1}\left(W_{\kappa, \mu+1}\right)\right\rangle_{\kappa, \mu+1},
\end{aligned}
$$

which shows that $\mathbf{L}_{m, \mathbf{k}}^{n}\left(W_{\kappa, \mu}\right)$ and $X_{d+1} \mathbf{L}_{m, \mathbf{k}}^{n-1}\left(W_{\kappa, \mu+1}\right)$ are elements of $\mathcal{V}_{n}\left(\mathbb{V}_{0}^{d+2}, \mathbf{w}_{\kappa}\right)$. Furthermore, it is easy to verify that

$$
\operatorname{dim} \mathcal{V}_{n}\left(\mathbb{V}_{0}^{d+2}, \mathrm{w}_{\kappa}\right)=\operatorname{dim} \mathcal{V}_{n}\left(\mathbb{V}^{d+1}, W_{\kappa, \mu}\right)+\operatorname{dim} \mathcal{V}_{n-1}\left(\mathbb{V}^{d+1}, W_{\kappa, \mu+1}\right)
$$

This completes the proof.

5.2. Reproducing and Poisson kernels. Let $\mathbf{P}_{n}\left(W_{\kappa, \mu} ; \cdot, \cdot\right)$ be the reproducing kerne of $\mathcal{V}_{n}\left(\mathbb{V}^{d+1}, W_{\kappa, \mu}\right)$. It is uniquely determined by

$$
\mathbf{b}_{\kappa, \mu} \int_{\mathbb{V}^{d+1}} P(y, s) \mathbf{P}_{n}\left(W_{\kappa, \mu} ; \cdot,(y, s)\right) W_{\kappa, \mu}(y, s) \mathrm{d} y \mathrm{~d} s=P, \quad \forall P \in \mathcal{V}_{n}\left(\mathbb{V}^{d+1}\left(W_{\kappa, \mu}\right) .\right.
$$

Theorem 5.4. Let $d \geq 1$ and $\mu \geq 0$. Let $\alpha=\alpha_{\kappa, \mu}=|\kappa|+\mu+\frac{d-1}{2}>0$. Then for $(x, t),(y, s) \in \mathbb{V}^{d+1}$,

$$
\begin{aligned}
& \mathbf{P}_{n}\left(W_{\kappa, \mu} ;(x, t),(y, s)\right)=C_{\kappa, \mu} \int_{[1,1]^{d+1}} \int_{0}^{\pi} L_{n}^{2 \alpha}(t+s+2 \boldsymbol{\rho}(x, t, y, s ; u) \cos \theta) \\
\times & e^{-\boldsymbol{\rho}(x, t, y, s ; u) \cos \theta} j_{\alpha-1}(\boldsymbol{\rho}(x, t, y, s ; u) \sin \theta)(\sin \theta)^{2 \alpha-1} \mathrm{~d} \theta \Phi_{\kappa}\left(u^{\prime}\right)\left(1-u_{d+1}^{2}\right)^{\mu-1} \mathrm{~d} u
\end{aligned}
$$

where $C_{\kappa}=\frac{2^{\alpha-1} \Gamma\left(\alpha+\frac{1}{2}\right)}{\sqrt{\pi}} c_{\kappa-\frac{1}{2}} c_{\mu-\frac{1}{2}}, u=\left(u^{\prime}, u_{d+1}\right)$ and

$$
\boldsymbol{\rho}(x, t, y, s ; u)=\sqrt{\frac{1}{2}\left(t s+x_{1} y_{1} u_{1}+\cdots+x_{d} y_{d} u_{d}+\sqrt{t^{2}-\|x\|^{2}} \sqrt{s^{2}-\|y\|^{2}} u_{d+1}\right)},
$$

and (5.5) holds under the limit (2.9) if either $\alpha=0$ or $\kappa_{i}=0$ for one or more $i$.

Proof. By Proposition 5.3 the space $\mathcal{V}_{n}\left(\mathbb{V}^{d+1}, W_{\kappa, \mu}\right)$ corresponds to the subspace of $\mathcal{V}_{n}\left(\mathbb{V}_{0}^{d+2}, \mathrm{w}_{\kappa}\right)$ that consists of polynomials even in $X_{d+1}$ variable. By symmetry, it follows readily that

$$
\mathbf{P}_{n}\left(W_{\kappa, \mu} ;(x, t),(y, s)\right)=\frac{1}{2}\left[\mathrm{P}_{n}\left(\mathrm{w}_{\kappa} ;(X, t),(Y, s)\right)+\mathrm{P}_{n}\left(\mathrm{w}_{\kappa} ;(X, t),\left(Y^{-}, s\right)\right)\right],
$$

where $\boldsymbol{\kappa}=\left(\kappa_{1}, \ldots, \kappa_{d+1}\right)$ and $\mathrm{P}_{n}\left(\mathrm{w}_{\kappa} ; \cdot, \cdot\right)$ is the reproducing kernel of $\mathcal{V}_{n}\left(\mathbb{V}^{d+2}, \mathrm{w}_{\kappa}\right)$. Consequently, (5.5) follows from (3.11) with $d+1$ replaced by $d+2$, where the integral with respect to $\mathrm{d} u_{d+1} \operatorname{losses}\left(1+u_{d+1}\right)$ factor because of the symmetry.

We can also state such a closed formula when $\alpha_{\kappa}=0$ by using (3.12). Since $\alpha_{\kappa}=0$ is equivalent to $\kappa=0, \mu=0$ and $d=1$, which is the degenerate case that is equivalent to the product Laguerre weight $w_{-\frac{1}{2}}\left(x_{1}\right) w_{-\frac{1}{2}}\left(x_{2}\right)$ on $\mathbb{R}_{+}^{2}$ by Remark 5.1, we shall not write down the formula.

We can also define the Poisson kernel of the orthogonal expansion on $\mathbb{V}^{d+1}$ by

$$
\mathbf{P}\left(W_{\kappa, \mu} ; r,(x, t),(y, s)\right)=\sum_{n=0}^{\infty} \mathbf{P}_{n}\left(W_{\kappa, \mu} ;(x, t),(y, s)\right) r^{n} .
$$

Then, as an analogue of Theorem 3.6. we obtain a closed form formula. 
Theorem 5.5. Under the same assumption as in Theorem 5.4.

$$
\begin{aligned}
\mathbf{P}\left(\mathrm{w}_{\kappa, \mu} ; r,(x, t),(y, s)\right)= & \frac{\mathrm{e}^{-\frac{(t+s) r}{1-r}}}{(1-r)^{2 \alpha_{\kappa, \mu}+1}} \int_{[-1,1]^{d+2}} \exp \left\{\frac{2 \sqrt{r} v}{1-r} \rho(x, t, y, s ; u)\right\} \\
& \times c_{\alpha_{\kappa, \mu}-\frac{1}{2}} c_{\kappa-\frac{1}{2}} \Phi_{\kappa}\left(u^{\prime}\right)\left(1-u_{d+1}^{2}\right)^{\mu-1} \mathrm{~d} u\left(1-v^{2}\right)^{\alpha_{\kappa, \mu}-1} \mathrm{~d} v,
\end{aligned}
$$

and it holds under the limit (2.9) if either $\alpha_{\kappa}=0$ or $\kappa_{i}=0$ for one or more $i$.

5.3. Pseudo convolution on the cone. We can also define a pseudo convolution on the solid cone $\mathbb{V}^{d+1}$, which will be bounded in the space $L_{\mathbf{u}(\kappa, \mu)}^{p}\left(\mathbb{V}^{d+1}\right)$, defined as the space of functions with finite $\|f\|_{p, \kappa, \mu}$ norm, where

$$
\|f\|_{p, \kappa, \mu}:=\left(\mathbf{b}_{\kappa, \mu} \int_{\mathbb{V}^{d+1}}\left|f(x, t) \mathrm{e}^{-t / 2}\right|^{p} \mathbf{u}(z, t) \mathrm{d} x \mathrm{~d} t\right)^{\frac{1}{p}},
$$

for $1 \leq p<\infty$ and $\mathbf{u}(x, t)=h_{\kappa}^{2}(x)\left(t^{2}-\|x\|^{2}\right)^{\mu-\frac{1}{2}}$, and

$$
\|f\|_{\infty}=\|f\|_{\infty, \kappa, \mu}:=\operatorname{ess} \sup \left\{f(x, t) \mathrm{e}^{-t / 2}:(x, t) \in \mathbb{V}^{d+1}\right\} .
$$

Using the notation of Theorem 5.4 with $\alpha=\alpha_{\kappa, \mu}>0$, we define, for $\in L^{1}\left(\mathbb{R}_{+}, \varpi_{2 \alpha_{\kappa, \mu}}\right)$ and $(x, y) \in \mathbb{V}^{d+1}$, the generalized translation operator

$$
\begin{aligned}
\mathbf{T}_{(x, t)} g(y, s)= & C_{\kappa, \mu} \int_{[-1,1]^{d+1}} \int_{0}^{\pi} g(t+s+2 \boldsymbol{\rho}(x, t, y, s ; u) \cos \theta) e^{-\boldsymbol{\rho}(x, t, y, s ; u) \cos \theta} \\
& \times j_{\alpha_{\kappa}-1}(\boldsymbol{\rho}(x, t, y, s ; u) \sin \theta)(\sin \theta)^{2 \alpha_{\kappa, \mu}-1} \mathrm{~d} \theta \Phi_{\kappa}\left(u^{\prime}\right)\left(1-u_{d+1}^{2}\right)^{\mu-1} \mathrm{~d} u .
\end{aligned}
$$

By its definition and (5.5), the reproducing kernel $\mathbf{P}_{n}\left(W_{k, \mu}\right)$ can be written as

$$
\mathbf{P}_{n}\left(W_{\kappa, \mu} ;(x, t), \cdot\right)=\mathbf{T}_{(x, t)} L_{n}^{2|\kappa|+2 \mu+d-1}, \quad n=0,1,2, \ldots,
$$

Proposition 5.6. Let $d \geq 1$ and $\alpha_{\kappa, \mu}=\mu+|\kappa|+\frac{d-1}{2} \geq \frac{1}{2}$. For $g \in L_{u\left(2 \alpha_{\kappa, \mu}\right)}^{p}\left(\mathbb{R}_{+}\right)$,

$$
\left\|\mathbf{T}_{(x, t)} g\right\|_{p, \kappa, \mu} \leq e^{t / 2}\|g\|_{p, u\left(2 \alpha_{\kappa}\right)}, \quad 1 \leq p \leq \infty .
$$

Proof. Let $\mathrm{T}_{(X, t)}$ denote the generalized translation on the conic surface $\mathbb{V}_{0}^{d+2}$, defined as in Definition 4.1 but with $\mathrm{w}_{\kappa}$ replaced by $\mathrm{w}_{\kappa}$ and $\rho(x, t, y, s ; u)$ replaced by $\boldsymbol{\rho}(x, t, y, s ; u)$. Comparing the definitions, it follows that

$$
\mathbf{T}_{(x, t)} g(y, s)=\frac{1}{2}\left[\mathrm{~T}_{(X, t)}(Y, s)+\mathrm{T}_{(X, t)}\left(Y^{-}, s\right)\right]
$$

and we also have $\left.\mathrm{T}_{(X, t)}\left(Y^{-}, s\right)=\mathrm{T}_{\left(X^{-}, t\right)}(Y, s)\right)$. Hence, by (5.4), the boundedness of $\mathbf{T}_{(x, t)}$ as stated follows from the boundedness of $\mathbf{T}_{(x, t)}$ in Proposition 4.2 .

For $\alpha_{\kappa, \mu}<\frac{1}{2}$, we have a counterpart of Proposition 4.4 .

Proposition 5.7. Let $d \geq 1$ and $\alpha_{\kappa, \mu} \leq \frac{1}{2}$. For $g \in L_{u\left(2 \alpha_{\kappa, \mu}\right)}^{1}\left(\mathbb{R}_{+}\right) \cap L_{u\left(2 \alpha_{\kappa, \mu}+\frac{1}{2}\right)}^{1}\left(\mathbb{R}_{+}\right)$,

$$
\left\|\mathbf{T}_{(x, t)} g\right\|_{1, \kappa, \mu} \leq e^{t / 2}\|g\|_{1, u\left(2 \alpha_{\kappa, \mu}\right)}+\sqrt{t} e^{t / 2}\|g\|_{1, u\left(2 \alpha_{\kappa, \mu}+\frac{1}{2}\right)}, \quad 1 \leq p \leq \infty .
$$

The generalized operator is used to defined a pseudo convolution operator on $\mathbb{V}^{d+1}$. For $f \in L_{\mathbf{u}(\kappa, \mu)}^{2}\left(\mathbb{V}^{d+1}\right)$ and $g \in L_{2 u\left(\alpha_{\kappa, \mu}\right)}^{2}\left(\mathbb{R}_{+}\right)$, we define

$$
f * \mathbb{V} g(x, t)=\mathbf{b}_{\kappa, \mu} \int_{\mathbb{V}^{d+1}} f(y, s) \mathbf{T}_{(x, t)} g(y, s) W_{\kappa, \mu}(y, s) \mathrm{d} y \mathrm{~d} s, \quad(x, t) \in \mathbb{V}^{d+1} .
$$


Theorem 5.8. Let $d \geq 1$ and $\alpha_{\kappa, \mu} \geq \frac{1}{2}$. For $f \in L_{\mathbf{u}(\kappa, \mu)}^{p}\left(\mathbb{V}^{d+1}\right), 1 \leq p \leq \infty$, and $g \in L_{u\left(2 \alpha_{\kappa, \mu}\right)}^{1}\left(\mathbb{R}_{+}\right)$,

$$
\|f * \mathbb{V} g\|_{p, \kappa, \mu} \leq\|f\|_{p, \kappa, \mu}\|g\|_{1, u\left(2 \alpha_{\kappa, \mu}\right)}, \quad 1 \leq p \leq \infty .
$$

Proof. Let $f * \mathbb{V}_{0} g$ denote the pseudo convolution on $\mathbb{V}_{0}^{d+2}$ defined as in Definition 4.5 with $\kappa$ replaced by $\kappa$ and $d+1$ replaced by $d+2$. By (5.7), it follows that

$$
f * \mathbb{V} g(x, t)=\frac{1}{2}\left[f * \mathbb{V}_{0} g(X, t)+f * \mathbb{V}_{0} g\left(X^{-}, t\right)\right]
$$

Hence, by (5.4), the boundedness of $f * \mathbb{V} g$ follows from the boundedness of $f * \mathbb{V}_{0} g$ in Theorem 4.7 .

Let the space $L_{\mathbf{u}(\kappa, \mu), *}^{p}\left(\mathbb{V}^{d+1}\right)$ be defined with an additional $\sqrt{t}$ in its norm $\|f\|_{p, \kappa, \mu}^{*}$, in analogous to the space $L_{\mathrm{u}(\kappa), *}^{p}\left(\mathbb{V}_{0}^{d+1}\right)$. We also have an analogue of Theorem 4.9.

Theorem 5.9. Let $d \geq 2$ and $\alpha_{\kappa, \mu}<\frac{1}{2}$. For $f \in L_{\mathbf{u}(\kappa, \mu)}^{1}\left(\mathbb{V}^{d+1}\right) \cap L_{\mathbf{u}(\kappa, \mu), *}^{1}\left(\mathbb{V}^{d+1}\right)$ and $g \in L_{u\left(2 \alpha_{\kappa, \mu}\right)}^{1}\left(\mathbb{R}_{+}\right) \cap L_{u\left(2 \alpha_{\kappa, \mu}+\frac{1}{2}\right)}^{1}\left(\mathbb{R}_{+}\right)$,

$$
\|f * \mathbb{V} g\|_{1, \kappa, \mu} \leq\|f\|_{1, \kappa, \mu}\|g\|_{1, u\left(2 \alpha_{\kappa, \mu}\right)}+\|f\|_{1, \kappa, \mu}^{*}\|g\|_{1, u\left(2 \alpha_{\kappa, \mu}+\frac{1}{2}\right)} .
$$

5.4. Cesàro means of Fourier-Laguerre series on the conic surface. For $\delta>$ -1 , the Cesàro means of the Fourier-Laguerre series is given by

$$
\mathbf{S}_{n}^{\delta}\left(W_{\kappa, \mu} ; f\right)=\frac{1}{\left(\begin{array}{c}
n+\delta \\
n
\end{array}\right)} \sum_{k=0}^{n}\left(\begin{array}{c}
n-k+\delta \\
n-k
\end{array}\right) \operatorname{proj}_{k}\left(W_{\kappa, \mu} ; f\right) .
$$

Theorem 5.10. Let $d \geq 1$ and $\mu \geq 0$. Assume $\alpha_{\kappa, \mu}=|\kappa|+\mu+\frac{d-1}{2} \geq \frac{1}{2}$. Then $\mathbf{S}_{n}^{\delta}\left(W_{\kappa, \mu} ; f\right)$ converse to $f$ in $L_{\mathbf{u}(\kappa, \mu)}^{p}\left(\mathbb{V}^{d+1}\right), 1 \leq p \leq \infty$, if $\delta>2|\kappa|+2 \mu+d-\frac{1}{2}$ and the inequality is sharp if $p=1$ and $p=\infty$.

Proof. As in the proof of Theorem 4.11] we need the boundedness of

$$
\left\|\mathbf{S}_{n}^{\delta}\left(W_{\kappa, \mu}\right)\right\|_{p, \kappa, \mu}=\int_{\mathbb{V}^{d}}\left|\mathbf{K}_{n}^{\delta}\left(W_{\kappa, \mu} ;(x, t),(y, s)\right)\right| \mathrm{e}^{s / 2} W_{\kappa, \mu}(y, s) \mathrm{d} y \mathrm{~d} s
$$

where $p=1$ or $p=\infty$ and $\mathbf{K}_{n}^{\delta}\left(W_{\kappa, \mu}\right)$ is the kernel of the $(C, \delta)$ means, which can be written in terms of its counterpart $\mathrm{K}_{n}^{\delta}\left(\mathrm{w}_{\boldsymbol{\kappa}}\right)$, with $\boldsymbol{\kappa}=(\kappa, \mu)$, on $\mathbb{V}_{0}^{d+2}$ as

$$
\mathbf{K}_{n}^{\delta}\left(W_{\kappa, \mu} ;(x, t),(y, s)\right)=\frac{1}{2}\left[\mathbf{K}_{n}^{\delta}\left(\mathbf{w}_{\kappa} ;(X, t),(Y, s)\right)+\mathbf{K}_{n}^{\delta}\left(\mathbf{w}_{\kappa} ;(X, t),\left(Y^{-}, s\right)\right)\right],
$$

so that the boundedness of $\left\|\mathbf{S}_{n}^{\delta}\left(W_{\kappa, \mu}\right)\right\|_{p, \kappa, \mu}$ from above follows from Theorem 4.11, Furthermore, since $\mathbf{K}_{n}^{\delta}\left(W_{\kappa, \mu} ;(x, t),(0,0)\right)=\mathbf{K}_{n}^{\delta}\left(\mathrm{w}_{\kappa} ;(X, t),(0,0)\right)$, the boundedness from below also follows by the proof of Theorem 4.11

For $\alpha_{\kappa, \mu} \leq \frac{1}{2}$, we can state the following counterpart of Theorem 4.12

Theorem 5.11. Let $d \geq 1$ and $\mu \geq 0$. Assume $\alpha_{\kappa, \mu} \leq \frac{1}{2}$. Then $\mathbf{S}_{n}^{\delta}\left(W_{\kappa, \mu} ; f\right)$ converse to $f$ in $L_{\mathbf{u}(\kappa, \mu)}^{1}\left(\mathbb{V}^{d+1}\right) \cap L_{\mathbf{u}(\kappa, \mu), *}^{1}\left(\mathbb{V}^{d+1}\right)$ if $\delta>2 \alpha_{\kappa, \mu}+1$. Moreover, $\mathbf{S}_{n}^{\delta}\left(W_{\kappa, \mu} ; f\right)$ does not converge for all $f \in L_{\mathbf{u}(\kappa, \mu)}^{1}\left(\mathbb{V}^{d+1}\right)$ if $\delta \leq 2 \alpha_{\kappa, \mu}+\frac{1}{2}$. 
As discussed in Remark [5.1, when $d=1$, our set-up is equivalent to the orthogonal expansions with respect to $U_{\kappa, \mu}$, defined in (5.2), on $\mathbb{R}_{+}^{2}$, which becomes product Laguerre expansions if $\kappa=0$; the case $\kappa>0$ has not been studied as far as we are aware. Let $L_{u(\kappa, \mu)}^{p}\left(\mathbb{R}_{+}^{2}\right)$ be the space with the norm

$$
\|f\|_{p, u(\kappa, \mu)}=\left(\int_{\mathbb{R}_{+}^{2}}\left|f\left(x_{1}, x_{2}\right)\right| \mathrm{e}^{\left(x_{1}+x_{2}\right) / 2} U_{\kappa, \mu}\left(x_{1}, x_{2}\right) \mathrm{d} x_{1} \mathrm{~d} x_{2}\right)^{\frac{1}{p}}, \quad 1 \leq p<\infty,
$$

and

$$
\|f\| \infty, u(\kappa, \mu)=\operatorname{ess} \sup \left\{\left|f\left(x_{1}, x_{2}\right)\right| \mathrm{e}^{-\left(x_{1}+x_{2}\right) / 2}:\left(x_{1}, x_{2}\right) \in \mathbb{R}_{+}^{2}\right\} .
$$

Let $S_{n}^{\delta}\left(U_{\kappa, \mu} ; f\right)$ be the Cesàro means of the Fourier-orthogonal expansions with respect to $U_{\kappa, \mu}$ on $\mathbb{R}_{+}^{2}$. Then our Theorem 5.10 for $d=2$ yields:

Corollary 5.12. Let $\kappa, \mu \geq 0$. If $\kappa+\mu \geq \frac{1}{2}$, then $S_{n}^{\delta}\left(U_{\kappa, \mu} ; f\right)$ converges to $f \in$ $L_{u(\kappa, \mu)}^{p}\left(\mathbb{R}_{+}^{2}\right), 1 \leq p \leq \infty$, if $\delta>2 \kappa+2 \mu+\frac{1}{2}$ and the result is sharp if $p=1$ or $p=\infty$.

If $\kappa+\mu<\frac{1}{2}$, we can also state a corollary of Theorem 5.11 that gives a sufficient condition $\delta>2 \kappa+2 \mu+1$ for the convergence in $L^{1}$ norm. For $\kappa=0$, the result for $\mu \geq \frac{1}{2}$ agrees with the product Lagueree expansions for $w_{\alpha}\left(x_{1}\right) w_{\beta}\left(x_{2}\right)$ with $\alpha=\beta=\mu-\frac{1}{2} \geq 0$ (cf. 21, Theorem 2.3]); while the result for $\mu<\frac{1}{2}$ provides a sufficient condition for the product Laguerre expansions with $\alpha=\beta<0$, which however is likely not sharp.

\section{REFERENCES}

[1] R. Askey and S. Wainger, Mean convergence of expansions in Laguerre and Hermite series, Amer. J. Math. 87 (1965), 695-708.

[2] P. Boggarapu, L. Roncal and S. Thangavelu, Mixed norm estimates for the Cesàro means associated with Dunkl-Hermite expansions. Trans. Amer. Math. Soc. 369 (2017), 7021-7047.

[3] NIST Digital Library of Mathematical Functions. http://dlmf.nist.gov/

[4] F. Dai and Y. Xu, Approximation theory and harmonic analysis on spheres and balls. Springer Monographs in Mathematics, Springer, 2013.

[5] C. F. Dunkl, Differential-difference operators associated to reflection groups. Trans. Amer. Math. Soc. 311 (1989), 167-183.

[6] C. F. Dunkl and Y. Xu, Orthogonal Polynomials of Several Variables Encyclopedia of Mathematics and its Applications 155, Cambridge University Press, Cambridge, 2014.

[7] E. Görlich and C. Markett, A convolution structure for Laguerre series, Indag. Math., 44 (1982), p. $161-171$.

[8] C. Markett, Mean Cesàro summability of Laguerre expansions and norm estimates with shifted parameter, Analysis Math., 8 (1982), 19-37.

[9] C. Meaney, Divergent Cesàro and Riesz means of Jacobi and Laguerre expansions. Proc. Amer. Math. Soc. 131 (2003), 3123-3128.

[10] B. Muckenhoupt and D. Webb, Two-Weight Norm Inequalities for Cesàro Means of Laguerre Expansions. Trans. Amer. Math. Soc. 353 (2001), 1119-1149.

[11] A. Nowak and K. Stempak, Riesz transforms for multi-dimensional Laguerre function expansions. Adv. Math. 215 (2007), 642-678.

[12] A. Nowak, K. Stempak and T. Szarek, On harmonic analysis operators in Laguerre-Dunkl and Laguerre-symmetrized settings. SIGMA 12 (2016), Paper No. 096, 39 pp.

[13] S. Olver and Y. Xu, Non-homogeneous wave equation on a cone. Integral Transforms Spec Funct., to appear.

[14] Eileen Poiani, Mean Cesàro summability of Laguerre and Hermite series, Trans. Amer. Math. Soc. 173 (1972), 1-31.

[15] R. Radha and S. Thangavelu, Hardy's inequalities for Hermite and Laguerre expansions. Proc. Am. Math. Soc. 132 (2004), 3525-3536.

[16] G. Szegö, Orthogonal polynomials. 4th edition, Amer. Math. Soc., Providence, RI. 1975

[17] S. Thangavelu, Summability of Laguerre expansions, Analysis Math. 16 (1990), 303-315. 
[18] S. Thangavelu, Lectures on Hermite and Laguerre Expansions, Princeton Univ. Press, Princeton, NJ, 1993.

[19] G. N. Watson, Another note on Laguerre polynomials, J. London Math. Soc., 14 (1939), 19-22.

[20] Y. Xu, Integration of the intertwining operator for h-harmonic polynomials associated to reflection groups. Proc. Amer. Math. Soc., 125 (1997), 2963-2973.

[21] Y. Xu, A note on summability of Laguerre expansions, Proc. Amer. Math. Soc., 128 (2000), 3571-3578.

[22] Y. Xu, Orthogonal polynomials and Fourier orthogonal series on a cone. J. Fourier Anal. Appl. 26 (2020), Article number:36

[23] Y. Xu, Orthogonal structure and orthogonal series in and on a double cone or a hyperboloid. Trans. Amer. Math. Soc. in print.

Department of Mathematics, University of Oregon, Eugene, Oregon 97403-1222.

Email address: yuan@uoregon.edu 